Research Square
Preprints are preliminary reports that have not undergone peer review.

They should not be considered conclusive, used to inform clinical practice, or referenced by the media as validated information.

\title{
Effects of HIV Status and History of Pregnancy Termination on Trends of Family Planning Knowledge among Women Living in Nine Fragile Countries
}

Daniel Woytowich ( $\nabla$ daniel.woytowich@cgu.edu )

Claremont Graduate University School of Community and Global Health

Bin Xie

Claremont Graduate University School of Community and Global Health

\section{Research Article}

Keywords: "fragile or conflict-affected situations (FCS)", "reproductive health", "family planning knowledge", "contraception knowledge", "sexual health", "pregnancy termination", "human immunodeficiency virus (HIV)", "Demographic and Health Surveys", "unmet need for contraception", "low- and middle-income countries (LMICs)"

Posted Date: September 8th, 2021

DOI: https://doi.org/10.21203/rs.3.rs-841640/v1

License: @ (i) This work is licensed under a Creative Commons Attribution 4.0 International License. Read Full License 


\section{Abstract}

Background: To decrease the proportion of women in fragile or conflict-affected situations (FCS) that need, but are not using modern contraception, the global health community must better understand family planning (FP) knowledge gaps; and elucidate which subgroups of women are, and are not, effectively being reached with FP information. This study investigated whether women with notable sexual and reproductive health histories that put them at risk for future pregnancy complications, namely HIV+ women and women with history of terminated pregnancy (HTP), would possess more complete FP knowledge and/or recall being given FP information more readily than HIV- women and women with no HTP (NHTP), respectively.

Methods: Knowledge of several contraceptive methods, and the prevalence of women who recalled being given FP information at a healthcare visit, or via TV, radio, or periodical were estimated across HIV, HTP, and country subgroups. HIV+ and HTP status were major predictors in multivariate logistic regressions analyzing the odds of knowing a specified method of contraception or of recalling being given FP information via the modes considered.

Results: HIV+ as opposed to HIV- women showed significantly higher odds of knowing about the pill $(p=0.001)$, IUD $(p<0.001)$, injectables $(p=0.005)$, male condoms $(p<0.001)$, female condoms $(p<0.001)$, and implants $(p<0.001)$; but not female or male sterilization. HIV+ women demonstrated higher odds of recalling being given FP information at a health visit $(p=0.001)$, whereas HTP women did not. HTP women showed higher odds ( $p<0.001)$ of knowing each modern method of FP with the exception of male sterilization and of recalling TV $(p=0.01)$ and radio $(p<0.001)$ FP messages.

Conclusions: Notable findings were the higher FP awareness among HIV+ and HTP women, and the observation that HIV+ women were seemingly provided FP information more readily at healthcare visits than were HTP women, despite their similar risk profiles concerning future pregnancies. Subsequent studies may elucidate why these patterns were observed and indicate which other diagnostic groups are effectively being reached with FP education, and which are not. Understanding trends and predictors of FP knowledge can help practitioners better target FP counseling efforts in FCS toward knowledge gaps and their causes.

\section{Background}

An estimated 1.8 billion people worldwide live in regions experiencing fragility or conflict, and this number is projected to be 2.3 billion by 2030 [1]. Despite the magnitude of this problem, health system strengthening in fragile or conflict-affected situations (FCS) is inadequately researched and donor investment is low, unless it is an area of strategic interest [2-4]. Therefore, it is essential for the public health community to continually work to better define health issues as they exist specifically in fragile regions. Doing so allows practitioners to create interventions that are better targeted, and whose downstream effects can be multiplied to effect change in a variety of societal spheres, which maximizes efficiency and cost-effectiveness. Such opportunities lie in the interrelated topics of reproductive health, sexual health, and family planning (FP), which have been recognized in recent decades as key issues in reaching not only women's health goals, but broader economic, educational, and developmental goals as well [5-10]. Reproductive and sexual health are also major components of equity and gender issues, which have been identified by stakeholders as areas in need of increased attention in FCS $[6,11,12]$.

Over a third of worldwide maternal deaths occur in FCS regions [13]. Other reproductive and sexual health outcomes like low birthweight babies, premature births, pregnancy terminations, and HIV transmission are common as well [13-17]. Roughly a third of worldwide AIDS patients reside in fragile regions [13]. As of 2019, 15.9 million women in sub-Saharan Africa (SSA), which contains several areas affected by fragility and conflict, were HIV+ [18]. Several research articles have reviewed the complex and sometimes counterintuitive relationship between conflict and HIV [19-26]. In 2015, the rate of pregnancies ending in stillbirth in SSA was 28.7 per 1000 births whereas in developed countries it was 3.4 per 1000, and the highest stillbirth rates were observed in conflict and emergency affected regions [27-29]. Many analyses in low- and middle-income countries (LMICs) regarding reproductive and sexual health have focused on trends of contraceptive use and unmet need [5,30-34], predictors of adverse pregnancy outcomes [35-38], and predictors of HIV infection [39-43]. However, investigations into levels of FP knowledge [44-47], which is a prerequisite for engaging in FP practices, are significantly less common. No projects that we are aware of have studied predictors of individual-level FP knowledge in an FCS multi-country study. Understanding how FP knowledge differs between subgroups of women, and elucidating determinants of that knowledge would play a modest, yet important role in helping to improve the efficacy of FP education and outreach efforts in fragile regions.

Due in part to the increased likelihood of subsequent morbidity they can present to the woman, her partners, and future offspring; HIV positivity and history of pregnancy termination (e.g., miscarriage, abortion, stillbirth) are issues that can lead to women in LMICs being targeted for sexual and reproductive health outreach. Therefore, we used Demographic and Health Surveys (DHS) [48] to assess whether HIV + women and women with a history of terminated pregnancy (HTP) would possess different FP knowledge profiles and/or recall being given or encountering FP information at differing rates than HIV- women and women with no history of terminated pregnancy (NHTP), respectively. We hypothesized that HIV + and HTP women would possess better FP knowledge profiles due to their presumed increased contact with health systems and outreach efforts, and the greater exposure to FP counseling that should accompany that contact. It is also feasible that they could be psychologically sensitized or more receptive to, and therefore more likely to remember FP messaging they receive or are exposed to, as opposed to HIV- and NHTP women.

Here, we report our study that was conducted on a sample of over 60,000 women of reproductive age living in fragile countries. In addition to HIV + and HTP status, DHS has individual-level data on relevant sociodemographic, reproductive health, and sexual health indicators along with several FP knowledge and messaging outcomes. The availability of this wide range of variables allowed for comprehensive subgroup analyses that took into account a variety of potential sociodemographic influences. This study can serve as a framework for future analyses on the prevalence and predictors of FP knowledge among vulnerable groups of women living in fragile regions. Subsequently, these efforts will aid practitioners and policymakers in better targeting FP information dissemination and resources toward not only existing gaps in FP knowledge, but the causes of those gaps as well.

\section{Methods}




\section{Data Sources}

Permission to use Standard DHS [48] and AIDS Indicator Surveys (AIS) [50] was obtained. The IPUMS [51] DHS data management tool was used to append the data to ensure maximum harmony, standardization, and comparability among variables and across countries. Country AISs were merged in afterwards since AIS data was not available through IPUMS. DHS data is from nationally representative household surveys that use a two-stage stratified cluster sampling design to collect cross-sectional data in LMICs. DHS surveys are implemented by regional agencies such as National Statistical Offices. Survey protocols were approved by both ICF International [52] and each country's institutional review board (IRB). This secondary analysis was approved by the Claremont Graduate University IRB. Advantages of DHS datasets include standardized data collection which allows for consistency across populations, national coverage, accurate representation of hard-to-reach groups due to complex sampling techniques, comprehensive training for interviewers, and typical response rates over $90 \%[49]$.

\section{Inclusion Criteria, Sampling, Weighting}

The most recent DHS surveys from nine fragile countries were analyzed. The included countries are Angola, Burundi, Cameroon, the Democratic Republic of Congo (DRC), Guinea, Cote d'Ivoire, Malawi, Niger, and Zimbabwe [53]. These countries were selected because they had accompanying AIS data, were available in DHS IPUMS, had all variables of interest, and had their most recent DHS survey conducted no earlier than 2011 (i.e., all information analyzed was no older than ten years). For the purposes of this study, countries were designated as fragile if they had appeared on the World Bank Group's (WBG) annually revised FCS list [54] at any time from 2011 and on. The WBG bases fragility status on metrics like Country Policy and Institutional Assessment (CPIA) scores [55], presence of UN peacekeeping forces, and numbers of refugees produced. CPIA scores are calculated using data collected by the WBG, African Development Bank, Asian Development Bank, UN Department of Peace Operations, and the UN High Commissioner for Refugees [54, 55]. Conflict status is based on conflict deaths as reported by the Armed Conflict Location and Event Data Project [56] and the Uppsala Conflict Data Program [57].

Respondents were women of child-bearing age (15-49 years). In the first stage of the sampling design, enumeration areas were drawn from census files according to population and stratification characteristics. In the second stage systematic sampling was used to select households from each enumeration area for interviews [48]. Respondents that consented to HIV testing provided a capillary blood sample for analysis [48, 50]. Since it was necessary to know HIV status for this analysis, only respondents that were tested for HIV and had valid results were included. Merging the multi-country DHS IPUMS dataset with each country's AIS yielded an initial sample with 130,780 cases. Cases that were not tested for HIV $(n=67,620)$ were eliminated, along with HIV results that were indeterminate $(n=8)$ or inconclusive $(n=254)$. Cases entered as missing for pregnancy termination were eliminated $(n=16)$. This resulted in a final dataset with 62,882 respondents. To ensure proper representation of population subgroups, weights were applied to adjust for the DHS complex survey design [58]. More information about sampling, weighting, and other DHS methodology can be found in the Guide to DHS Statistics, DHS-7, Version 2 [58].

\section{Variables}

Major Predicting Variables: HIV status (HIV+, HIV-) and pregnancy termination status (HTP, NHTP) were the major predicting variables. Respondents' history of pregnancy termination was ascertained by interviewers asking: "Have you ever had a pregnancy that miscarried, was aborted, or ended in stillbirth?" Answers were coded as 'no (0)' or 'yes (1)'. HIV status was obtained from the blood test done as part of the AIS. Results were either 'no (0)', meaning the respondent was HIV negative; or 'yes (1)', indicating the respondent tested positive for HIV1 and/or HIV2.

Outcome Variables: There were a total of 15 distinct, dichotomous outcome variables in the analysis, each recorded as 'yes (1)' or 'no (0)'. Categories of FP knowledge considered were whether the respondent had awareness of modern methods of FP, including the pill (i.e., birth control pill), intrauterine device (IUD), injectables (e.g., Depo-Provera), male condoms, female condoms, female sterilization, male sterilization, and implants (e.g., Norplant). Traditional methods of FP knowledge considered were awareness of withdrawal (i.e., withdrawal of the penis before ejaculation) and the rhythm method (i.e., periodic abstinence at the high-fertility part of the menstrual cycle). Also included was a variable regarding whether or not they knew 'at least one modern method of FP', where 'yes' meant the respondent knew at least one of the aforementioned modern methods, and 'no' meant they only knew a traditional method, folkloric method (e.g., herbs, amulet), or no method at all. Lastly, there were four FP messaging outcome variables. These variables described whether a respondent: a) recalled being given FP information by a provider at a visit to a health facility (of those who had visited a health facility for any reason in the last year), b) recalled encountering a FP message on TV in recent months, $\mathbf{c}$ ) recalled hearing a FP message on the radio in recent months, and/or $\mathbf{d}$ ) recalled reading a FP message in a periodical in recent months.

\section{Covariates}

Ten covariates were included in the predictive model based on data availability and epidemiological plausibility as indicated by relevant literature [59-62]. They include country of residence, age (15-22, 23-32, 33-49 years), highest education level completed (none, primary, secondary, higher), wealth index quintile (poorest, poorer, middle, richer, richest), urban/rural status, whether the respondent was employed (yes, no), marital status (never married, currently married, formerly married), whether the respondent's home has electricity (yes, no), whether the respondent owns a mobile phone (yes, no), and whether the respondent considers herself to be the primary FP decider in her household (yes, no). DHS calculates wealth index by principal component analysis which places households on a continuous scale of relative wealth according to assets reflective of living standard, such as flooring material, drinking water source, type of toilet facility used, etc. A respondent was considered to be 'employed' if they had worked outside the home during the last 12 months and was paid with cash or in-kind payments.

\section{Statistical Analyses}

The prevalence of HIV + and HTP were determined across country, sociodemographic, reproductive health, and sexual health categories. Percentages of women who knew about each method of FP in subgroup analyses based on country, HIV status, and HTP status were calculated along with $95 \%$ confidence 
intervals $(\mathrm{Cl})$ and significance levels based on second order Rao-Scott design-adjusted chi-square ( $\mathrm{X} 2)$ values. A multivariate logistic regression model was employed which included the aforementioned major predictors and covariates and each FP knowledge or messaging category as a dichotomous outcome variable. Adjusted odds ratios (AOR) were calculated and corresponding 95\% Cls reflect their precision and significance. HIV-and NHTP women were reference groups. Therefore, any OR greater than one (1) signified higher odds that an HIV + or HTP woman would possess a category of FP knowledge or recall receiving FP messaging via one of the modes investigated. Statistical analyses were completed using IBM SPSS Version 26 [63] and sample weights were applied using the IBM SPSS Complex Samples package according to the procedures outlined in the Guide to DHS Statistics, DHS-7, Version 2 [58].

\section{Results \\ Overview}

The nine countries included in the study are in SSA and shown in red in Fig. 1 below. The weighted percentage of the sample who were HIV + was 5.5\% (95\% $\mathrm{Cl}=5.3-5.8)$. The weighted percentage of women who had HTP was $14.2 \%(95 \% \mathrm{Cl}=13.9-14.6)$. The mean age of respondents was 28.2 years, and $64.3 \%$ were married. The country that contributed the most cases was the DRC $(n=9313,14.8 \%)$, whereas the country that contributed the least was Cote $d^{\prime}$ Ivoire ( $n$ $=4655,7.3 \%$ ).

\section{Descriptive Results}

Table 1 shows the counts and percentages of respondents from each country, the year of the DHS, and each country's HIV + and HTP prevalence. P-values and $95 \%$ Cls were estimated so the statistical significance of differences across all categories of a variable (e.g., across all five subgroups of the wealth index variable) and between specific variable subcategories (e.g., the 'poorer' wealth index quintile compared to the 'richest' group) could be assessed, respectively. The prevalence of HIV + ranged from $0.4 \%$ in Niger $(\mathrm{Cl}=0.2-0.5)$ to $16.7 \%$ in Zimbabwe $(\mathrm{Cl}=15.6-17.8)$ while the prevalence of HTP ranged from $9.8 \%$ in Guinea $(\mathrm{Cl}=8.8-11.0)$ to $23.5 \%$ in Cameroon $(\mathrm{Cl}=22.4-24.7)$. Table 1 provides the prevalence of HIV + and HTP across sociodemographic characteristics in the form of row percentages. For example, $9.1 \%(\mathrm{Cl}=8.6-9.6)$ of all women aged 33-49 were $\mathrm{HIV}+$, whereas $23.4 \%(\mathrm{Cl}=22.7-24.1)$ had $\mathrm{HTP}$. Urban residents had higher rates of $\mathrm{HIV}+(6.7 \%, \mathrm{Cl}=6.2-7.2)$ and $\mathrm{HTP}(15.2 \%, \mathrm{Cl}=14.6-15.9)$ compared to rural residents $(\mathrm{p}<0.001)$. Respondents with a secondary education showed the highest prevalence of $\mathrm{HIV}+(7.1 \%, \mathrm{Cl}=6.6-7.6)$; however, this was not a significant difference from the primary $(6.5 \%, \mathrm{Cl}=6.1-7.0)$ and higher education $(6.2 \%, \mathrm{Cl}=5.0-7.7)$ groups. Women with no education had the highest prevalence of HTP $(15.8 \%, \mathrm{Cl}=15.1-16.5)$; but this was not significantly different from the primary education $(14.9 \%, \mathrm{Cl}=14.3-15.5)$ or higher education $(14.1 \%, \mathrm{Cl}=12.4-16.1)$ groups. Literate women showed a higher prevalence $(\mathrm{p}<0.001)$ of $\mathrm{HIV}+(7.0 \%, \mathrm{Cl}=6.6-7.4)$ compared to illiterate women $(3.3 \%, \mathrm{Cl}=3.1-3.6)$; whereas illiterate women had a higher prevalence $(\mathrm{p}<$ $0.001)$ of $\operatorname{HTP}(15.2 \%, \mathrm{Cl}=14.6-15.8)$ than literate women $(13.6 \%, \mathrm{Cl}=13.1-14.1)$. Table 1 also includes HIV + and HTP prevalence across wealth index, employment, electricity and communication, marriage, head of household, and recent healthcare visit variables. 
Table 1

Prevalence of HIV and history of terminated pregnancy across sociodemographic variables

\begin{tabular}{|c|c|c|c|c|c|}
\hline Sociodemographic Variables & $\begin{array}{l}\mathrm{n}(\% \text { of } \\
\text { total) }\end{array}$ & HIV Positive & $\begin{array}{l}\chi^{2} \\
\text { (P-Value) }\end{array}$ & $\begin{array}{l}\text { History of Terminated } \\
\text { Pregnancy }\end{array}$ & $\begin{array}{l}\chi^{2} \\
\text { (P-Value) }\end{array}$ \\
\hline & & n (row \%, $95 \% \mathrm{Cl}$ ) & & n (row \%, $95 \% \mathrm{Cl}$ ) & \\
\hline Country (year of DHS) & & & $\begin{array}{l}243.0(P< \\
0.001)\end{array}$ & & $57.8(P<0.001)$ \\
\hline Angola (2015-16) & $\begin{array}{l}6620 \\
(10.4)\end{array}$ & $193(2.6,2.1-3.2)$ & & $612(11.2,10.0-12.6)$ & \\
\hline Burundi(2016-17) & $\begin{array}{l}8492 \\
(13.6)\end{array}$ & $119(1.2,0.9-1.5)$ & & $1088(13.4,12.6-14.3)$ & \\
\hline Cameroon (2011) & $\begin{array}{l}7243 \\
(11.5)\end{array}$ & $433(5.6,5.0-6.3)$ & & $1671(23.5,22.4-24.7)$ & \\
\hline Dem. Rep. Congo (2013-14) & $\begin{array}{l}9313 \\
(14.8)\end{array}$ & $133(1.6,1.2-2.2)$ & & $1337(14.1,13.0-15.2)$ & \\
\hline Guinea (2012) & $4687(7.5)$ & $108(2.1,1.7-2.6)$ & & $514(9.8,8.8-11.0)$ & \\
\hline Cote d'Ivoire (2011-12) & $4655(7.3)$ & $209(4.6,3.9-5.4)$ & & $883(19.8,18.1-21.5)$ & \\
\hline Malawi (2016) & $\begin{array}{l}7718 \\
(12.3)\end{array}$ & $\begin{array}{l}858(11.0,10.1- \\
12.0)\end{array}$ & & $780(10.7,9.8-11.7)$ & \\
\hline Niger (2012) & $5100(8.2)$ & $27(0.4,0.2-0.5)$ & & $714(14.7,13.5-16.0)$ & \\
\hline Zimbabwe (2015) & $\begin{array}{l}9054 \\
(14.4)\end{array}$ & $\begin{array}{l}1583(16.7,15.6- \\
17.8)\end{array}$ & & $1051(12.2,11.4-13.0)$ & \\
\hline Age & & & $\begin{array}{l}338.2(P< \\
0.001)\end{array}$ & & $\begin{array}{l}1008.6(P< \\
0.001)\end{array}$ \\
\hline $15-22$ years & $\begin{array}{l}21553 \\
(34.2)\end{array}$ & $447(2.0,1.7-2.2)$ & & $990(4.7,4.4-5.1)$ & \\
\hline $23-32$ years & $\begin{array}{l}21246 \\
(34.0)\end{array}$ & $1322(5.9,5.5-6.3)$ & & $3076(15.2,14.6-15.9)$ & \\
\hline $33-49$ years & $\begin{array}{l}20083 \\
(31.7)\end{array}$ & $1894(9.1,8.6-9.6)$ & & $4584(23.4,22.7-24.1)$ & \\
\hline Urban/Rural & & & $\begin{array}{l}42.6(P< \\
0.001)\end{array}$ & & $14.3(P<0.001)$ \\
\hline Urban & $\begin{array}{l}23616 \\
(35.6)\end{array}$ & $1681(6.7,6.2-7.2)$ & & $3350(15.2,14.6-15.9)$ & \\
\hline Rural & $\begin{array}{l}39266 \\
(64.4)\end{array}$ & $1982(4.9,4.6-5.2)$ & & $5300(13.7,13.2-14.1)$ & \\
\hline Highest Achieved Education & & & $\begin{array}{l}90.9(P< \\
0.001)\end{array}$ & & $23.0(P<0.001)$ \\
\hline None & $\begin{array}{l}18567 \\
(29.3)\end{array}$ & $510(2.6,2.3-2.9)$ & & $2858(15.8,15.1-16.5)$ & \\
\hline Primary & $\begin{array}{l}21055 \\
(33.5)\end{array}$ & $1413(6.5,6.1-7.0)$ & & $3041(14.9,14.3-15.5)$ & \\
\hline Secondary & $\begin{array}{l}21223 \\
(33.7)\end{array}$ & $1596(7.1,6.6-7.6)$ & & $2474(12.2,11.7-12.8)$ & \\
\hline Higher & $2027(3.5)$ & $144(6.2,5.0-7.7)$ & & $277(14.1,12.4-16.1)$ & \\
\hline Literate & & & $\begin{array}{l}228.4(P< \\
0.001)\end{array}$ & & $18.3(P<0.001)$ \\
\hline Yes & $\begin{array}{l}37806 \\
(60.6)\end{array}$ & $2810(7.0,6.6-7.4)$ & & $4924(13.6,13.1-14.1)$ & \\
\hline No & $\begin{array}{l}25001 \\
(39.4)\end{array}$ & $851(3.3,3.1-3.6)$ & & $3715(15.2,14.6-15.8)$ & \\
\hline Wealth Index Quintile & & & $\begin{array}{l}11.4(\mathrm{P}< \\
0.001)\end{array}$ & & $2.44(P=0.045)$ \\
\hline Poorest & $\begin{array}{l}11433 \\
(18.1)\end{array}$ & $557(4.7,4.2-5.1)$ & & $1476(13.3,12.6-14.2)$ & \\
\hline Poorer & $\begin{array}{l}11946 \\
(18.9)\end{array}$ & $549(4.5,4.0-4.9)$ & & $1632(13.8,13.1-14.6)$ & \\
\hline
\end{tabular}

Page 5/20 


\begin{tabular}{|c|c|c|c|c|c|}
\hline Sociodemographic Variables & $\begin{array}{l}\mathrm{n}(\% \text { of } \\
\text { total) }\end{array}$ & HIV Positive & $\begin{array}{l}\chi^{2} \\
\text { (P-Value) }\end{array}$ & $\begin{array}{l}\text { History of Terminated } \\
\text { Pregnancy }\end{array}$ & $\begin{array}{l}\chi^{2} \\
\text { (P-Value) }\end{array}$ \\
\hline Middle & $\begin{array}{l}12161 \\
(19.4)\end{array}$ & $681(5.4,4.9-6.0)$ & & $1694(14.4,13.6-15.2)$ & \\
\hline Richer & $\begin{array}{l}12936 \\
(20.7)\end{array}$ & $898(6.4,5.8-7.2)$ & & $1830(14.4,13.7-15.3)$ & \\
\hline Richest & $\begin{array}{l}14406 \\
(23.0)\end{array}$ & $978(6.3,5.8-6.9)$ & & $2018(15.0,14.2-15.8)$ & \\
\hline Currently Working & & & $\begin{array}{l}0.058(P= \\
0.81)\end{array}$ & & $\begin{array}{l}309.5(P< \\
0.001)\end{array}$ \\
\hline Yes & $\begin{array}{l}37775 \\
(60.0)\end{array}$ & $2197(5.6,5.2-5.9)$ & & $6165(16.9,16.3-17.4)$ & \\
\hline No & $\begin{array}{l}25018 \\
(40.0)\end{array}$ & $1463(5.5,5.2-5.9)$ & & $2475(10.3,9.8-10.8)$ & \\
\hline \multicolumn{6}{|l|}{ Electric/Communication } \\
\hline Has Electricity & $\begin{array}{l}19142 \\
(30.6)\end{array}$ & $1410(6.7,6.2-7.2)$ & $\begin{array}{l}38.0(P< \\
0.001)\end{array}$ & $2928(16.2,15.4-16.9)$ & $42.3(\mathrm{P}<0.001)$ \\
\hline No Electricity & $\begin{array}{l}42504 \\
(69.4)\end{array}$ & $2149(4.9,4.6-5.3)$ & & $5568(13.4,13.0-13.9)$ & \\
\hline Has Mobile Phone & $\begin{array}{l}41846 \\
(67.0)\end{array}$ & $2897(6.6,6.2-6.9)$ & $\begin{array}{l}147.5(P< \\
0.001)\end{array}$ & $5970(14.8,14.4-15.3)$ & $20.8(P<0.001)$ \\
\hline No Mobile Phone & $\begin{array}{l}21013 \\
(33.0)\end{array}$ & $766(3.4,3.1-3.8)$ & & $2676(13.0,12.4-13.7)$ & \\
\hline Has Radio & $\begin{array}{l}31196 \\
(50.9)\end{array}$ & $1682(5.1,4.8-5.4)$ & $\begin{array}{l}10.9(P= \\
0.001)\end{array}$ & $4483(14.9,14.4-15.4)$ & $12.5(P<0.001)$ \\
\hline No Radio & $\begin{array}{l}30453 \\
(49.1)\end{array}$ & $1878(5.9,5.5-6.3)$ & & $4011(13.6,13.1-14.1)$ & \\
\hline Has Television & $\begin{array}{l}19061 \\
(30.9)\end{array}$ & $1406(6.9,6.4-7.4)$ & $\begin{array}{l}57.1(P< \\
0.001)\end{array}$ & $2895(16.0,15.3-16.7)$ & $38.7(\mathrm{P}<0.001)$ \\
\hline No Television & $\begin{array}{l}42585 \\
(69.1)\end{array}$ & $2154(4.9,4.6-5.2)$ & & $5602(13.5,13.1-13.9)$ & \\
\hline Marriage Status & & & $\begin{array}{l}553.9(P< \\
0.001)\end{array}$ & & $\begin{array}{l}715.2(P< \\
0.001)\end{array}$ \\
\hline Never Married & $\begin{array}{l}16724 \\
(26.4)\end{array}$ & $490(2.7,2.4-3.0)$ & & $455(2.8,2.5-3.2)$ & \\
\hline Currently Married & $\begin{array}{l}40320 \\
(64.3)\end{array}$ & $2146(5.1,4.8-5.4)$ & & $7177(18.4,17.9-18.9)$ & \\
\hline Formerly Married & $5838(9.3)$ & $\begin{array}{l}1027(16.9,15.7- \\
18.2)\end{array}$ & & $1018(18.0,16.8-19.2)$ & \\
\hline Head of Household $(\mathrm{HH})$ & & & $\begin{array}{l}545.3(P< \\
0.001)\end{array}$ & & $35.1(P<0.001)$ \\
\hline Identified herself as $\mathrm{HH}$ & $\begin{array}{l}10206 \\
(15.7)\end{array}$ & $\begin{array}{l}1258(11.6,10.9- \\
12.5)\end{array}$ & & $1632(16.8,15.8-17.8)$ & \\
\hline Said other family member is $\mathrm{HH}$ or did not know & $\begin{array}{l}52673 \\
(84.3)\end{array}$ & $2405(4.4,4.2-4.7)$ & & $7018(13.8,13.4-14.2)$ & \\
\hline $\begin{array}{l}\text { Visited health facility for any reason in past } 12 \\
\text { months? }\end{array}$ & & & $\begin{array}{l}62.6(P< \\
0.001)\end{array}$ & & $\begin{array}{l}350.1(P< \\
0.001)\end{array}$ \\
\hline Yes & $\begin{array}{l}32564 \\
(53.0)\end{array}$ & $2224(6.4,6.1-6.8)$ & & $5485(17.3,16.8-17.9)$ & \\
\hline No & $\begin{array}{l}30300 \\
(47.0)\end{array}$ & $1438(4.5,4.2-4.9)$ & & $3163(10.8,10.3-11.2)$ & \\
\hline
\end{tabular}

Table 2 provides the prevalence of HIV + and HTP across key reproductive and sexual health variables. Like Table 1, row percentages are given for HIV + and HTP. Women who were pregnant at the time of the interview had an HIV + prevalence of $3.5 \%(\mathrm{Cl}=3.0-4.1)$, which was lower $(p<0.001)$ than the HIV + prevalence in women who were not pregnant $(5.8 \%, \mathrm{Cl}=5.5-6.0)$. Current pregnancy status had no significance in relation to prevalence of HTP $(p=0.144)$. Women who had not given birth in the five years leading up to the survey had the highest prevalence of HIV+ $(6.7 \%, 6.3-7.1)$ compared to women with one 
birth (5.8\%, $\mathrm{Cl}=5.4-6.3)$; two births $(3.2 \%, \mathrm{Cl}=2.8-3.6)$; three $(1.8 \%, \mathrm{Cl}=1.3-2.6)$, and four to six births $(2.4 \%, \mathrm{Cl}=0.7-7.7)$. Women with one birth in the previous 5 years had the highest prevalence of $\operatorname{HTP}(16.4 \%, \mathrm{Cl}=15.8-17.1)$. Out of women using contraception, women who identified themselves as the sole FP decision maker in their household had the lowest prevalence of $\mathrm{HIV}+(5.5 \%, \mathrm{Cl}=4.4-6.9)$, whereas women whose partner made the FP decisions had an $\mathrm{HIV}+$ rate of $9.5 \%(\mathrm{Cl}=7.9-11.3)$. In contrast, women who identified themselves as the sole FP decision maker had the highest prevalence of $\mathrm{HTP}(21.4 \%$, $\mathrm{Cl}=$ 19.1-23.9), but this was not a significant difference from those whose partner made FP decisions $(17.9 \%, \mathrm{Cl}=15.9-20.2)$. Out of women with an unmet need for contraception, $4.4 \%(\mathrm{Cl}=4.0-5.0)$ were HIV + whereas $15.3 \%(\mathrm{Cl}=14.5-16.2)$ had HTP. Table 2 also includes HIV + and HTP prevalence across variables indicating who has the final say on the respondent's healthcare decisions, the respondent's ability to refuse sex, age at first marriage/cohabitation, number of sex partners in the last 12 months, and condom use during last intercourse. Due to the large amount of information in Tables 1 and 2, all their results could not be described in the text, but the reader is encouraged to use p-values and Cls in the manner described above to observe patterns across other variables of interest. 
Table 2

Prevalence of HIV and history of terminated pregnancy across reproductive and sexual health variables

\begin{tabular}{|c|c|c|c|c|c|}
\hline Reproductive \& Sexual Health Variables & $\begin{array}{l}\text { n (\% of total } \\
\text { sample) }\end{array}$ & HIV Positive & $\begin{array}{l}\chi^{2} \\
\text { (P-Value) }\end{array}$ & $\begin{array}{l}\text { History of Terminated } \\
\text { Pregnancy }\end{array}$ & $\begin{array}{l}\chi^{2} \\
\text { (P-Value) }\end{array}$ \\
\hline & & $\begin{array}{l}\text { n (row \%, } 95 \% \\
\text { Cl) }\end{array}$ & & n (row \%, $95 \% \mathrm{Cl})$ & \\
\hline Pregnancy Status & & & $\begin{array}{l}41.2(P< \\
0.001)\end{array}$ & & $\begin{array}{l}2.14(P= \\
0.144)\end{array}$ \\
\hline Pregnant at time of interview & $6081(9.7)$ & $\begin{array}{l}225(3.5,3.0- \\
4.1)\end{array}$ & & $929(15.0,13.9-16.1)$ & \\
\hline Not Pregnant/Unsure & $56801(90.3)$ & $\begin{array}{l}3438(5.8,5.5- \\
6.0)\end{array}$ & & $7721(14.2,13.8-14.6)$ & \\
\hline Number of births in last 5 years & & & $\begin{array}{l}48.6(P< \\
0.001)\end{array}$ & & $\begin{array}{l}23.4(P< \\
0.001)\end{array}$ \\
\hline None & $27845(44.2)$ & $\begin{array}{l}1969(6.7,6.3- \\
7.1)\end{array}$ & & $3456(12.6,12.1-13.1)$ & \\
\hline One & $20134(32.0)$ & $\begin{array}{l}1223(5.8,5.4- \\
6.3)\end{array}$ & & $3142(16.4,15.8-17.1)$ & \\
\hline Two & $12719(20.4)$ & $\begin{array}{l}423(3.2,2.8- \\
3.6)\end{array}$ & & $1800(14.7,13.9-15.5)$ & \\
\hline Three & $2036(3.2)$ & $\begin{array}{l}44(1.8,1.3- \\
2.6)\end{array}$ & & $234(12.5,10.8-14.4)$ & \\
\hline 4 to 6 & $148(0.2)$ & $4(2.4,0.7-7.7)$ & & $18(14.2,7.9-24.2)$ & \\
\hline \multicolumn{6}{|l|}{ Who makes FP decisions? } \\
\hline Respondent & $1610(2.5)$ & $\begin{array}{l}103(5.5,4.4- \\
6.9)\end{array}$ & $\begin{array}{l}6.67(P= \\
0.001)\end{array}$ & $322(21.4,19.1-23.9)$ & $\begin{array}{l}5.88(P= \\
0.003)\end{array}$ \\
\hline Respondent's Partner & $1835(3.0)$ & $\begin{array}{l}180(9.5,7.9- \\
11.3)\end{array}$ & & $300(17.9,15.9-20.2)$ & \\
\hline Joint/Other/Don't Know & $8231(13.6)$ & $\begin{array}{l}701(8.1,7.4- \\
8.9)\end{array}$ & & $1346(17.0,16.0-18.1)$ & \\
\hline Not currently using contraception & $51119(80.9)$ & $\begin{array}{l}2675(5.0,4.7- \\
5.2)\end{array}$ & - & $6662(13.4,13.0-13.8)$ & - \\
\hline \multicolumn{6}{|c|}{$\begin{array}{l}\text { Who has the final say on the woman's healthcare } \\
\text { decisions? }\end{array}$} \\
\hline Respondent Alone & $6219(9.9)$ & $\begin{array}{l}561 \\
9.5)\end{array}(8.5,7.7-$ & $\begin{array}{l}60.0(P< \\
0.001)\end{array}$ & $1173(19.1,18.0-20.4)$ & $\begin{array}{l}3.73(P= \\
0.01)\end{array}$ \\
\hline Respondent + Partner (joint) & $15639(24.8)$ & $\begin{array}{l}941(5.8,5.3- \\
6.2)\end{array}$ & & $2727(17.9,17.2-18.7)$ & \\
\hline Respondent's Partner Alone & $17939(28.7)$ & $\begin{array}{l}617(3.4,3.0- \\
3.7)\end{array}$ & & $3210(18.7,18.0-19.4)$ & \\
\hline Someone else/Relatives & $454(0.7)$ & $\begin{array}{l}24(5.2,3.1- \\
8.3)\end{array}$ & & $56(12.5,9.2-16.6)$ & \\
\hline Not currently in partnership & $22564(35.7)$ & $\begin{array}{l}1519(6.3,5.9- \\
6.8)\end{array}$ & - & $1473(6.8,6.3-7.2)$ & - \\
\hline \multicolumn{6}{|l|}{ Can Respondent Refuse Sex? } \\
\hline Yes & $24191(38.7)$ & $\begin{array}{l}1499(5.8,5.5- \\
6.2)\end{array}$ & $\begin{array}{l}23.6(P< \\
0.001)\end{array}$ & $4653(20.1,19.4-20.8)$ & $\begin{array}{l}35.1(\mathrm{P}< \\
0.001)\end{array}$ \\
\hline No & $14781(23.6)$ & $\begin{array}{l}607(4.0,3.6- \\
4.5)\end{array}$ & & $2329(15.8,15.1-16.5)$ & \\
\hline Don't Know/Depends & $1292(1.9)$ & $\begin{array}{l}35(2.9,1.9- \\
4.4)\end{array}$ & & $179(15.1,12.6-18.0)$ & \\
\hline Neither married or in relationship & $22562(35.7)$ & $\begin{array}{l}1517(6.3,5.9- \\
6.8)\end{array}$ & - & $1473(6.8,6.3-7.2)$ & - \\
\hline Age at First Marriage/ Cohabitation & & & $\begin{array}{l}71.7(P< \\
0.001)\end{array}$ & & $\begin{array}{l}276.2(\mathrm{P}< \\
0.001)\end{array}$ \\
\hline Never Married & $16724(26.4)$ & $\begin{array}{l}490(2.7,2.4- \\
3.0)\end{array}$ & & $455(2.8,2.5-3.2)$ & \\
\hline
\end{tabular}




\begin{tabular}{|c|c|c|c|c|c|}
\hline Reproductive \& Sexual Health Variables & $\begin{array}{l}\mathrm{n} \text { (\% of total } \\
\text { sample) }\end{array}$ & HIV Positive & $\begin{array}{l}\mathrm{X}^{2} \\
\text { (P-Value) }\end{array}$ & $\begin{array}{l}\text { History of Terminated } \\
\text { Pregnancy }\end{array}$ & $\begin{array}{l}\chi^{2} \\
\text { (P-Value) }\end{array}$ \\
\hline $8-10$ years & $175(0.3)$ & $\begin{array}{l}13(7.2,4.0- \\
12.7)\end{array}$ & & $38(21.2,15.1-28.9)$ & \\
\hline $11-13$ years & $3236(5.3)$ & $\begin{array}{l}173(5.1,4.3- \\
6.1)\end{array}$ & & $594(18.7,17.1-20.4)$ & \\
\hline $14-16$ years & $13611(21.8)$ & $\begin{array}{l}766(5.4,4.9- \\
5.9)\end{array}$ & & $2253(16.9,16.1-17.7)$ & \\
\hline $17-19$ years & $15227(24.5)$ & $\begin{array}{l}1005(6.4,5.9- \\
6.9)\end{array}$ & & $2620(17.6,16.8-18.3)$ & \\
\hline 20-29 years & $12945(20.4)$ & $\begin{array}{l}1061(7.8,7.2- \\
8.4)\end{array}$ & & $2445(20.0,19.1-20.9)$ & \\
\hline $30-48$ years & $964(1.5)$ & $\begin{array}{l}155(16.4,13.6- \\
19.6)\end{array}$ & & $245(27.6,24.2-31.3)$ & \\
\hline Unmet Need for Contraception & & & $\begin{array}{l}86.3(P< \\
0.001)\end{array}$ & & $\begin{array}{l}61.7(P< \\
0.001)\end{array}$ \\
\hline Never had sex & $8241(13.0)$ & $\begin{array}{l}93(1.1,0.9- \\
1.4)\end{array}$ & & - & \\
\hline Unmet need for spacing/limiting & $11807(18.5)$ & $\begin{array}{l}535(4.4,4.0- \\
5.0)\end{array}$ & & $1751(15.3,14.5-16.2)$ & \\
\hline No unmet need/using & $30180(48.5)$ & $\begin{array}{l}2001(6.3,5.9- \\
6.7)\end{array}$ & & $4874(16.9,16.3-17.4)$ & \\
\hline Not married/no sex in last 30 days & $5454(8.8)$ & $\begin{array}{l}557(9.4,8.5- \\
10.3)^{-}\end{array}$ & & $558(10.1,9.2-11.1)$ & \\
\hline Menopausal/Infecund & $7148(11.1)$ & $\begin{array}{l}476(6.4,5.8- \\
7.1)\end{array}$ & & $1462(21.0,19.8-22.2)$ & \\
\hline $\begin{array}{l}\text { \# of sex partners in last } 12 \text { months (including } \\
\text { spouse) }\end{array}$ & & & $\begin{array}{l}22.9(P< \\
0.001)\end{array}$ & & $\begin{array}{l}275.9(P< \\
0.001)\end{array}$ \\
\hline None & $16729(26.5)$ & $\begin{array}{l}798(4.5,4.1- \\
4.9)\end{array}$ & & $923(5.6,5.2-6.1)$ & \\
\hline One & $44770(71.4)$ & $\begin{array}{l}2721(5.8,5.5- \\
6.1)\end{array}$ & & $7370(17.1,16.6-17.5)$ & \\
\hline 2 to 5 & $1271(2.0)$ & $\begin{array}{l}116(8.6,6.9- \\
10.7)\end{array}$ & & $339(27.7,24.8-30.7)$ & \\
\hline 6 to 9 & $22(0.0)$ & $0(0.0)$ & & $7(40.6,18.8-68.8)$ & \\
\hline 10 or more & $90(0.1)$ & $\begin{array}{l}28(23.6,15.6- \\
34.0)\end{array}$ & & $11(11.0,5.5-20.7)$ & \\
\hline \multirow[t]{2}{*}{$\begin{array}{l}\text { Used condom for most recent intercourse? (If in last } \\
12 \text { months) }\end{array}$} & & \multirow{2}{*}{\multicolumn{2}{|c|}{$\begin{array}{l}628.3(P< \\
0.001)\end{array}$}} & & 35.7 \\
\hline & & & & & $(P<0.001)$ \\
\hline Yes & $4818(10.4)$ & $\begin{array}{l}818(15.8,14.5- \\
17.2)\end{array}$ & & $609(13.5,12.4-14.8)$ & \\
\hline No & $41256(89.6)$ & $\begin{array}{l}2031(4.7,4.5- \\
5.0)\end{array}$ & & $7112(17.8,17.3-18.3)$ & \\
\hline
\end{tabular}

Table 3 provides the percentage of respondents in each country with knowledge of each category of FP method and who had recalled receiving FP information at a healthcare facility (if visited in the last 12 months), via TV, radio, or in a periodical in recent months. Table 3 differs from Tables 1 and 2 because it presents column percentages. The percentage of respondents who were familiar with 'at least one modern method' of FP ranged from $78.5 \%$ of the total sample from Angola $(\mathrm{Cl}=76.7-80.2)$ to $99.1 \%$ of the total sample from Zimbabwe $(\mathrm{Cl}=98.8-99.3)$. Respondents that knew about the pill ranged from $55.2 \%$ in the $\mathrm{DRC}(\mathrm{Cl}=52.4-58.1)$ to $97.4 \%$ in Zimbabwe $(\mathrm{Cl}=96.9-97.8)$. IUD knowledge ranged from $18.3 \%$ in Niger $(\mathrm{Cl}=16.5-20.1)$ to $91.7 \%$ in $\mathrm{Burundi}(\mathrm{Cl}$ $=90.9-92.3)$. Knowledge of injectables ranged from $56.9 \%$ in Angola $(\mathrm{Cl}-54.7-59.0)$ to $95.9 \%$ in Malawi $(\mathrm{Cl}=95.2-96.5)$. Male condom knowledge ranged from $50.8 \%$ in Niger $(\mathrm{Cl}=47.6-54.0)$ to $97.4 \%$ in Zimbabwe $(\mathrm{Cl}=96.9-97.8)$. Knowledge of female condoms ranged from $13.4 \%$ in Niger $(\mathrm{Cl}=11.9-15.0)$ to $92.9 \%$ in Malawi $(\mathrm{Cl}=92.1-93.6)$. Female sterilization knowledge ranged from $26.6 \%$ in Angola $(\mathrm{Cl}=24.5-28.9)$ to $88.7 \%$ in $\mathrm{Malawi}(\mathrm{Cl}=87.7-89.6)$. Knowledge of male sterilization ranged from $6.0 \%$ in Niger $(\mathrm{Cl}=5.2-7.0)$ to $66.5 \%$ in Malawi $(\mathrm{Cl}=64.9-68.0)$. Knowledge about implants ranged from $16.9 \%$ in Cote d'Ivoire $(\mathrm{Cl}=15.0-19.0)$ to $93.8 \%$ in Burundi $(\mathrm{Cl}=93.1-94.4)$. Of the women who had visited a health facility in the year prior to the survey, the percentage that recalled being given $\mathrm{FP}$ information while at that visit ranged from $11.2 \%$ in $\mathrm{Guinea}(\mathrm{Cl}=9.2-13.5)$ to $45.6 \%$ in $\mathrm{Malawi}(\mathrm{Cl}=43.5-47.8)$. Respondents who recalled seeing a FP message on TV in recent months ranged from 3.1\% in Burundi $(\mathrm{Cl}=2.6-3.7)$ to $28.0 \%$ in $\mathrm{Ang}$ ola $(\mathrm{Cl}=25.4-30.8)$. 
Those who recalled hearing a FP message on the radio ranged from $9.9 \%$ in the $\mathrm{DRC}(\mathrm{Cl}=7.8-12.4)$ to $43.0 \%$ in Niger $(\mathrm{Cl}=40.2-45.8)$. Women who recalled reading a FP message in a periodical ranged from $1.3 \%$ in Niger $(\mathrm{Cl}=1.0-1.7)$ to $19.8 \%$ in $\mathrm{Zimbabwe}(\mathrm{Cl}=18.5-21.3)$.

Table 3

Prevalence across countries of family planning knowledge and messaging received

\begin{tabular}{|c|c|c|c|c|c|c|c|c|c|}
\hline $\begin{array}{l}\text { Family Planning Knowledge and Messaging } \\
\text { Variables }\end{array}$ & Angola & Burundi & Cameroon & $\begin{array}{l}\text { Dem. } \\
\text { Rep. of } \\
\text { Congo }\end{array}$ & Guinea & $\begin{array}{l}\text { Cote } \\
\text { d'Ivoire }\end{array}$ & Malawi & Niger & Zimbabwe \\
\hline & $\begin{array}{l}\text { column } \\
\%(95 \% \\
\mathrm{Cl})\end{array}$ & $\begin{array}{l}\text { column } \\
\%(95 \% \\
\mathrm{Cl})\end{array}$ & $\begin{array}{l}\text { column \% } \\
(95 \% \mathrm{Cl})\end{array}$ & $\begin{array}{l}\text { column } \\
\%(95 \% \\
\mathrm{Cl})\end{array}$ & $\begin{array}{l}\text { column } \\
\%(95 \% \\
\mathrm{Cl})\end{array}$ & $\begin{array}{l}\text { column } \\
\%(95 \% \\
\mathrm{Cl})\end{array}$ & $\begin{array}{l}\text { column } \\
\%(95 \% \\
\mathrm{Cl})\end{array}$ & $\begin{array}{l}\text { column } \\
\%(95 \% \\
\mathrm{Cl})\end{array}$ & $\begin{array}{l}\text { column \% } \\
(95 \% \mathrm{Cl})\end{array}$ \\
\hline Knows at least one modem method of FP?* & $\begin{array}{l}78.5 \\
(76.7- \\
80.2)\end{array}$ & $\begin{array}{l}97.4 \\
(97.0- \\
97.7)\end{array}$ & $\begin{array}{l}93.3 \\
(91.7- \\
94.7)\end{array}$ & $\begin{array}{l}87.3 \\
(85.6- \\
88.9)\end{array}$ & $\begin{array}{l}89.5 \\
(88.1- \\
90.8)\end{array}$ & $\begin{array}{l}92.4 \\
(90.8- \\
93.7)\end{array}$ & $\begin{array}{l}98.4 \\
(97.9- \\
98.7)\end{array}$ & $\begin{array}{l}87.6 \\
(85.9- \\
89.1)\end{array}$ & $\begin{array}{l}99.1 \\
(98.8- \\
99.3)\end{array}$ \\
\hline \multicolumn{10}{|l|}{ Modern Methods of FP } \\
\hline Knows about pill* & $\begin{array}{l}61.0 \\
(58.7- \\
63.2)\end{array}$ & $\begin{array}{l}92.6 \\
(91.8- \\
93.3)\end{array}$ & $\begin{array}{l}74.1 \\
(72.2- \\
75.9)\end{array}$ & $\begin{array}{l}55.2 \\
(52.4- \\
58.1)\end{array}$ & $\begin{array}{l}78.4 \\
(76.1- \\
80.7)\end{array}$ & $\begin{array}{l}84.8 \\
(82.6- \\
86.7)\end{array}$ & $\begin{array}{l}93.4 \\
(92.6- \\
94.1)\end{array}$ & $\begin{array}{l}82.4 \\
(80.3- \\
84.3)\end{array}$ & $\begin{array}{l}97.4 \\
(96.9- \\
97.8)\end{array}$ \\
\hline Knows about IUD* & $\begin{array}{l}36.6 \\
(34.1- \\
39.3)\end{array}$ & $\begin{array}{l}91.7 \\
(90.9- \\
92.3)\end{array}$ & $\begin{array}{l}38.0 \\
(36.3- \\
39.7)\end{array}$ & $\begin{array}{l}23.5 \\
(21.7- \\
25.4)\end{array}$ & $\begin{array}{l}24.3 \\
(21.9- \\
26.8)\end{array}$ & $\begin{array}{l}24.8 \\
(22.4- \\
27.3)\end{array}$ & $\begin{array}{l}84.0 \\
(82.9- \\
85.0)\end{array}$ & $\begin{array}{l}18.3 \\
(16.5- \\
20.1)\end{array}$ & $\begin{array}{l}75.0 \\
(73.5- \\
76.4)\end{array}$ \\
\hline Knows about injectables* & $\begin{array}{l}56.9 \\
(54.7- \\
59.0)\end{array}$ & $\begin{array}{l}92.8 \\
(91.9- \\
93.5)\end{array}$ & $\begin{array}{l}75.1 \\
(73.3- \\
76.9)\end{array}$ & $\begin{array}{l}60.9 \\
(58.1- \\
63.5)\end{array}$ & $\begin{array}{l}78.2 \\
(75.8- \\
80.5)\end{array}$ & $\begin{array}{l}65.1 \\
(62.4- \\
67.8)\end{array}$ & $\begin{array}{l}95.9 \\
(95.2- \\
96.5)\end{array}$ & $\begin{array}{l}71.7 \\
(69.0- \\
74.2)\end{array}$ & $\begin{array}{l}95.4 \\
(94.8- \\
96.0)\end{array}$ \\
\hline Knows about male condoms* & $\begin{array}{l}72.2 \\
(70.1- \\
74.3)\end{array}$ & $\begin{array}{l}93.2 \\
(92.6- \\
93.9)\end{array}$ & $\begin{array}{l}89.8 \\
(88.0- \\
91.3)\end{array}$ & $\begin{array}{l}81.0 \\
(78.7- \\
83.1)\end{array}$ & $\begin{array}{l}76.7 \\
(73.8- \\
79.4)\end{array}$ & $\begin{array}{l}88.3 \\
(86.3- \\
90.1)\end{array}$ & $\begin{array}{l}96.9 \\
(96.4- \\
97.3)\end{array}$ & $\begin{array}{l}50.8 \\
(47.6- \\
54.0)\end{array}$ & $\begin{array}{l}97.4 \\
(96.9- \\
97.8)\end{array}$ \\
\hline Knows about female condoms* & $\begin{array}{l}49.5 \\
(46.8- \\
52.2)\end{array}$ & $\begin{array}{l}79.7 \\
(78.5- \\
80.9)\end{array}$ & $\begin{array}{l}69.0 \\
(67.0- \\
70.9)\end{array}$ & $\begin{array}{l}41.6 \\
(38.5- \\
44.7)\end{array}$ & $\begin{array}{l}28.3 \\
(25.5- \\
31.2)\end{array}$ & $\begin{array}{l}52.9 \\
(49.8- \\
56.0)\end{array}$ & $\begin{array}{l}92.9 \\
(92.1- \\
93.6)\end{array}$ & $\begin{array}{l}13.4 \\
(11.9- \\
15.0)\end{array}$ & $\begin{array}{l}92.7 \\
(91.9- \\
93.4)\end{array}$ \\
\hline Knows about female sterilization* & $\begin{array}{l}26.6 \\
(24.5- \\
28.9)\end{array}$ & $\begin{array}{l}78.5 \\
(77.2- \\
79.8)\end{array}$ & $\begin{array}{l}44.6 \\
(42.7- \\
46.4)\end{array}$ & $\begin{array}{l}51.9 \\
(49.4- \\
54.4)\end{array}$ & $\begin{array}{l}41.2 \\
(38.2- \\
44.3)\end{array}$ & $\begin{array}{l}28.5 \\
(26.1- \\
31.1)\end{array}$ & $\begin{array}{l}88.7 \\
(87.7- \\
89.6)\end{array}$ & $\begin{array}{l}32.2 \\
(29.4- \\
35.0)\end{array}$ & $\begin{array}{l}59.1 \\
(57.4- \\
60.8)\end{array}$ \\
\hline Knows about male sterilization* & $\begin{array}{l}17.7 \\
(16.0- \\
19.6)\end{array}$ & $\begin{array}{l}61.2 \\
(59.7- \\
62.7)\end{array}$ & $\begin{array}{l}15.8 \\
(14.7- \\
17.1)\end{array}$ & $\begin{array}{l}19.4 \\
(17.8- \\
21.1)\end{array}$ & $\begin{array}{l}13.3 \\
(11.1- \\
15.8)\end{array}$ & $\begin{array}{l}14.4 \\
(12.7- \\
16.2)\end{array}$ & $\begin{array}{l}66.5 \\
(64.9- \\
68.0)\end{array}$ & $\begin{array}{l}6.0 \\
(5.2- \\
7.0)\end{array}$ & $\begin{array}{l}37.1 \\
(35.5- \\
38.8)\end{array}$ \\
\hline Knows about implants like Norplant* & $\begin{array}{l}48.8 \\
(46.2- \\
51.5)\end{array}$ & $\begin{array}{l}93.8 \\
(93.1- \\
94.4)\end{array}$ & $\begin{array}{l}45.1 \\
(43.2- \\
46.9)\end{array}$ & $\begin{array}{l}28.5 \\
(25.6- \\
31.7)\end{array}$ & $\begin{array}{l}20.8 \\
(18.7- \\
23.0)\end{array}$ & $\begin{array}{l}16.9 \\
(15.0- \\
19.0)\end{array}$ & $\begin{array}{l}92.3 \\
(91.4- \\
93.1)\end{array}$ & $\begin{array}{l}23.1 \\
(21.1- \\
25.3)\end{array}$ & $\begin{array}{l}91.1 \\
(90.2- \\
91.8)\end{array}$ \\
\hline \multicolumn{10}{|l|}{ Traditional Methods of FP } \\
\hline Knows about withdrawal* & $\begin{array}{l}34.3 \\
(31.9- \\
36.9)\end{array}$ & $\begin{array}{l}62.7 \\
(61.2- \\
64.2)\end{array}$ & $\begin{array}{l}51.4 \\
(49.6- \\
53.3)\end{array}$ & $\begin{array}{l}60.2 \\
(57.8- \\
62.6)\end{array}$ & $\begin{array}{l}38.0 \\
(35.1- \\
41.0)\end{array}$ & $\begin{array}{l}43.1 \\
(40.0- \\
46.2)\end{array}$ & $\begin{array}{l}70.7 \\
(69.1- \\
72.4)\end{array}$ & $\begin{array}{l}13.5 \\
(12.0- \\
15.1)\end{array}$ & $\begin{array}{l}70.4 \\
(69.1- \\
71.7)\end{array}$ \\
\hline Knows rhythm method* & $\begin{array}{l}35.9 \\
(33.5- \\
38.4)\end{array}$ & $\begin{array}{l}57.7 \\
(56.2- \\
59.3)\end{array}$ & $\begin{array}{l}65.6 \\
(63.5- \\
67.7)\end{array}$ & $\begin{array}{l}64.2 \\
(61.8- \\
66.6)\end{array}$ & $\begin{array}{l}44.4 \\
(41.5- \\
47.4)\end{array}$ & $\begin{array}{l}48.8 \\
(45.9- \\
51.7)\end{array}$ & $\begin{array}{l}63.8 \\
(62.3- \\
65.3)\end{array}$ & $\begin{array}{l}10.9 \\
(9.6- \\
12.5)\end{array}$ & $\begin{array}{l}42.7 \\
(41.2- \\
44.2)\end{array}$ \\
\hline \multicolumn{10}{|l|}{ FP Messaging Received } \\
\hline $\begin{array}{l}\text { Heard/saw FP TV message in recent months? } \\
\star\end{array}$ & $\begin{array}{l}28.0 \\
(25.4- \\
30.8)\end{array}$ & $\begin{array}{l}3.1 \\
(2.6- \\
3.7)\end{array}$ & $\begin{array}{l}22.7 \\
(21.0- \\
24.5)\end{array}$ & $\begin{array}{l}4.8(3.9- \\
6.0)\end{array}$ & $\begin{array}{l}10.9 \\
(9.3- \\
12.9)\end{array}$ & $\begin{array}{l}22.1 \\
(20.1- \\
24.3)\end{array}$ & $\begin{array}{l}10.4 \\
(9.2- \\
11.7)\end{array}$ & $\begin{array}{l}12.9 \\
(11.3- \\
14.5)\end{array}$ & $\begin{array}{l}17.3 \\
(15.9- \\
18.7)\end{array}$ \\
\hline Heard FP radio message in recent months?* & $\begin{array}{l}25.2 \\
(22.8- \\
27.7)\end{array}$ & $\begin{array}{l}30.3 \\
(28.9- \\
31.7)\end{array}$ & $\begin{array}{l}18.3 \\
(16.8- \\
19.9)\end{array}$ & $\begin{array}{l}9.9 \\
(7.8- \\
12.4)\end{array}$ & $\begin{array}{l}28.7 \\
(25.8- \\
31.7)\end{array}$ & $\begin{array}{l}15.1 \\
(13.6- \\
16.8)\end{array}$ & $\begin{array}{l}41.3 \\
(39.7- \\
43.0)\end{array}$ & $\begin{array}{l}43.0 \\
(40.2- \\
45.8)\end{array}$ & $\begin{array}{l}28.2 \\
(26.8- \\
29.6)\end{array}$ \\
\hline $\begin{array}{l}\text { Read FP message in periodical in recent } \\
\text { months?* }\end{array}$ & $\begin{array}{l}10.2 \\
(8.5- \\
12.2)\end{array}$ & $\begin{array}{l}2.9 \\
(2.5- \\
3.4)\end{array}$ & $\begin{array}{l}10.4(9.3- \\
11.7)\end{array}$ & $\begin{array}{l}2.3 \\
(1.7- \\
3.1)\end{array}$ & $\begin{array}{l}3.2 \\
(2.5- \\
4.0)\end{array}$ & $\begin{array}{l}5.3 \\
(4.3- \\
6.4)\end{array}$ & $\begin{array}{l}9.7 \\
(8.7- \\
10.9)\end{array}$ & $\begin{array}{l}1.3 \\
(1.0- \\
1.7)\end{array}$ & $\begin{array}{l}19.8 \\
(18.5- \\
21.3)\end{array}$ \\
\hline $\begin{array}{l}\text { Proportion who visited a health facility in last } \\
\text { year }\end{array}$ & $\begin{array}{l}45.4 \\
(43.1- \\
47.7)\end{array}$ & $\begin{array}{l}73.7 \\
(72.3- \\
75.0)\end{array}$ & $\begin{array}{l}53.7 \\
(51.8- \\
55.6)\end{array}$ & $\begin{array}{l}38.9 \\
(36.2- \\
41.7)\end{array}$ & $\begin{array}{l}44.4 \\
(41.9- \\
47.0)\end{array}$ & $\begin{array}{l}46.8 \\
(44.4- \\
49.2)\end{array}$ & $\begin{array}{l}61.5 \\
(59.7- \\
63.2)\end{array}$ & $\begin{array}{l}48.5 \\
(46.2- \\
50.9)\end{array}$ & $\begin{array}{l}56.0 \\
(54.3- \\
57.7)\end{array}$ \\
\hline $\begin{array}{l}\text { Out of those who had visited a health facility, } \\
\text { how many recalled being given FP info by } \\
\text { provider?* }\end{array}$ & $\begin{array}{l}28.5 \\
(25.4- \\
31.8)\end{array}$ & $\begin{array}{l}35.7 \\
(34.0- \\
37.4)\end{array}$ & $\begin{array}{l}24.3 \\
(22.3- \\
26.4)\end{array}$ & $\begin{array}{l}21.2 \\
(18.5- \\
24.2)\end{array}$ & $\begin{array}{l}11.2 \\
(9.2- \\
13.5)\end{array}$ & $\begin{array}{l}19.7 \\
(17.2- \\
22.3)\end{array}$ & $\begin{array}{l}45.6 \\
(43.5- \\
47.8)\end{array}$ & $\begin{array}{l}23.5 \\
(21.2- \\
26.0)\end{array}$ & $\begin{array}{l}39.8 \\
(37.9- \\
41.7)\end{array}$ \\
\hline
\end{tabular}


Table 4 shows the differences between HIV + and HIV-, and between HTP and NHTP women with respect to the FP knowledge and messaging categories. Column percentages are presented. The second column of Table 4 shows the percentage of the total sample that reported knowledge of the corresponding FP category or who had received FP information via one of the modes considered. The modern FP method that the highest percentage of respondents were aware of was male condoms (84.8\%); followed by the pill (80.0\%), injectables (78.2\%), female condoms (61.9\%), implants (56.4\%), female sterilization (53.5\%), IUD $(50.5 \%)$, and male sterilization (30.0\%). Out of women who visited a healthcare facility in the last 12 months, $30.7 \%$ recalled being given FP information by a provider. Out of the whole sample, $13.9 \%, 26.2 \%$, and $7.8 \%$ recalled hearing or seeing a FP message via the TV, radio, or periodical, respectively. Columns three and four show the percentage and Cls of HIV + versus HIV- women aware of each category of FP and who had recalled receiving FP messaging via the modes considered. Corresponding p-values are in column five. Columns six and seven show the percentage and Cls of HTP versus NHTP women with knowledge of each category of FP and who had been reached with each mode of FP messaging. Corresponding p-values are in column eight. 
Table 4

Family planning knowledge and messaging variables across HIV and history of terminated pregnancy subgroups

\begin{tabular}{|c|c|c|c|c|c|c|c|}
\hline $\begin{array}{l}\text { Family Planning Knowledge and Messaging } \\
\text { Variables }\end{array}$ & $\begin{array}{l}n= \\
62882\end{array}$ & $\begin{array}{l}\text { HIV } \\
\text { Positive, n } \\
=3663\end{array}$ & $\begin{array}{l}\text { HIV } \\
\text { Negative, } \\
n=59219\end{array}$ & $\begin{array}{l}\chi^{2} \\
\text { (P- } \\
\text { Value) }\end{array}$ & $\begin{array}{l}\text { History of } \\
\text { Terminated } \\
\text { Pregnancy, } \\
\mathrm{n}=\mathbf{8 6 5 0}\end{array}$ & $\begin{array}{l}\text { No History of } \\
\text { Terminated Pregnancy, } \\
n=54232\end{array}$ & $\begin{array}{l}x^{2} \\
(\mathrm{P}- \\
\text { Value })\end{array}$ \\
\hline & $\begin{array}{l}\mathrm{n}(\% \text { of } \\
\text { total } \\
\text { sample) }\end{array}$ & $\begin{array}{l}\text { n (column } \\
\%, 95 \% \\
\text { Cl) }\end{array}$ & $\begin{array}{l}\text { n (column } \\
\%, 95 \% \mathrm{Cl})\end{array}$ & & $\begin{array}{l}\text { n (column } \\
\%, 95 \% \mathrm{Cl})\end{array}$ & n (column \%, $95 \% \mathrm{Cl})$ & \\
\hline Knows at least one modem method of FP? & $\begin{array}{l}57314 \\
(92.1)\end{array}$ & $\begin{array}{l}3565 \\
(97.7 \\
96.9- \\
98.3)\end{array}$ & $\begin{array}{l}53749 \\
(91.8, \\
91.4-92.2)\end{array}$ & $\begin{array}{l}81.1 \\
(P< \\
0.001)\end{array}$ & $\begin{array}{l}8254(96.0 \\
95.5-96.5)\end{array}$ & $\begin{array}{l}49060(91.4,91.0- \\
91.9)\end{array}$ & $\begin{array}{l}151.5 \\
(P< \\
0.001)\end{array}$ \\
\hline \multicolumn{8}{|l|}{ Modern methods of FP } \\
\hline Knows about pill & $\begin{array}{l}49317 \\
(80.0)\end{array}$ & $\begin{array}{l}3391 \\
(93.1 \\
91.7- \\
94.2)\end{array}$ & $\begin{array}{l}45926 \\
(79.2, \\
78.6-79.9)\end{array}$ & $\begin{array}{l}174.3 \\
(P< \\
0.001)\end{array}$ & $\begin{array}{l}7442(87.3 \\
86.3-88.2)\end{array}$ & $\begin{array}{l}41875(78.7,78.1- \\
79.4)\end{array}$ & $\begin{array}{l}218.5 \\
(P< \\
0.001)\end{array}$ \\
\hline Knows about IUD & $\begin{array}{l}30901 \\
(50.5)\end{array}$ & $\begin{array}{l}2657 \\
(73.1 \\
71.1- \\
75.0)\end{array}$ & $\begin{array}{l}28244 \\
(49.3 \\
48.5-50.0)\end{array}$ & $\begin{array}{l}441.4 \\
(P< \\
0.001)\end{array}$ & $\begin{array}{l}4762(56.5 \\
55.1-58.0)\end{array}$ & $\begin{array}{l}26139(49.4,48.6- \\
50.2)\end{array}$ & $\begin{array}{l}94.3(P \\
< \\
0.001)\end{array}$ \\
\hline Knows about injectables & $\begin{array}{l}48119 \\
(78.2)\end{array}$ & $\begin{array}{l}3341 \\
(91.8 \\
90.2- \\
93.2)\end{array}$ & $\begin{array}{l}44778 \\
(77.5, \\
76.8-78.1)\end{array}$ & $\begin{array}{l}154.4 \\
(\mathrm{P}< \\
0.001)\end{array}$ & $\begin{array}{l}7384(86.5 \\
85.5-87.4)\end{array}$ & $\begin{array}{l}40735(76.8,76.1- \\
77.5)\end{array}$ & $\begin{array}{l}270.1 \\
(P< \\
0.001)\end{array}$ \\
\hline Knows about male condoms & $\begin{array}{l}52847 \\
(84.8)\end{array}$ & $\begin{array}{l}3511 \\
(96.4 \\
95.5- \\
97.1)\end{array}$ & $\begin{array}{l}49336 \\
(84.3 \\
83.6-84.9)\end{array}$ & $\begin{array}{l}226.0 \\
(P< \\
0.001)\end{array}$ & $\begin{array}{l}7706(89.5 \\
88.6-90.4)\end{array}$ & $\begin{array}{l}45141(84.0,83.4- \\
84.6)\end{array}$ & $\begin{array}{l}101.8 \\
(\mathrm{P}< \\
0.001)\end{array}$ \\
\hline Knows about female condoms & $\begin{array}{l}38036 \\
(61.9)\end{array}$ & $\begin{array}{l}3133 \\
(86.7 \\
85.0-88.2)\end{array}$ & $\begin{array}{l}34903 \\
(60.7 \\
59.8-61.5)\end{array}$ & $\begin{array}{l}511.9 \\
(P< \\
0.001)\end{array}$ & $\begin{array}{l}5628(67.0 \\
65.6-68.4)\end{array}$ & $\begin{array}{l}32408(61.1,60.2- \\
61.9)\end{array}$ & $\begin{array}{l}68.5(P \\
< \\
0.001)\end{array}$ \\
\hline Knows about female sterilization & $\begin{array}{l}32838 \\
(53.5)\end{array}$ & $\begin{array}{l}2361 \\
(65.1 \\
63.0- \\
67.0)\end{array}$ & $\begin{array}{l}30477 \\
(53.0, \\
52.2-53.7)\end{array}$ & $\begin{array}{l}124.3 \\
(P< \\
0.001)\end{array}$ & $\begin{array}{l}5102(59.7 \\
58.3-61.1)\end{array}$ & $\begin{array}{l}27736(52.5,51.7- \\
53.2)\end{array}$ & $\begin{array}{l}103.8 \\
(\mathrm{P}< \\
0.001)\end{array}$ \\
\hline Knows about male sterilization & $\begin{array}{l}19047 \\
(30.9)\end{array}$ & $\begin{array}{l}1524 \\
(41.7 \\
39.6- \\
43.9)\end{array}$ & $\begin{array}{l}17523 \\
(30.4, \\
29.8-31.0)\end{array}$ & $\begin{array}{l}114.3 \\
(P< \\
0.001)\end{array}$ & $\begin{array}{l}2706(31.7 \\
30.4-33.0)\end{array}$ & $\begin{array}{l}16341(30.8,30.2- \\
31.5)\end{array}$ & $\begin{array}{l}1.64(P \\
= \\
0.200)\end{array}$ \\
\hline Knows about implants like Norplant & $\begin{array}{l}34590 \\
(56.4)\end{array}$ & $\begin{array}{l}2952 \\
(81.3 \\
79.4- \\
83.1)\end{array}$ & $\begin{array}{l}31638 \\
(55.0, \\
54.2-55.8)\end{array}$ & $\begin{array}{l}479.5 \\
(P< \\
0.001)\end{array}$ & $\begin{array}{l}5097(60.8 \\
59.3-62.2)\end{array}$ & $\begin{array}{l}29493(55.7,54.8- \\
56.5)\end{array}$ & $\begin{array}{l}48.6(P \\
< \\
0.001)\end{array}$ \\
\hline \multicolumn{8}{|l|}{ Traditional methods of FP } \\
\hline Knows about withdrawal & $\begin{array}{l}32681 \\
(52.9)\end{array}$ & $\begin{array}{l}2560 \\
(71.4 \\
69.5- \\
73.3)\end{array}$ & $\begin{array}{l}30121 \\
(52.0, \\
51.2-52.7)\end{array}$ & $\begin{array}{l}332.8 \\
(P< \\
0.001)\end{array}$ & $\begin{array}{l}5454(64.0 \\
62.6-65.4)\end{array}$ & $\begin{array}{l}27227(51.0,50.2- \\
51.8)\end{array}$ & $\begin{array}{l}304.4 \\
(P< \\
0.001)\end{array}$ \\
\hline Knows the rhythm method & $\begin{array}{l}31300 \\
(50.4)\end{array}$ & $\begin{array}{l}2154 \\
(59.0 \\
57.0-61.1)\end{array}$ & $\begin{array}{l}29146 \\
(50.2, \\
49.4-51.0)\end{array}$ & $\begin{array}{l}63.95 \\
(\mathrm{P}< \\
0.001)\end{array}$ & $\begin{array}{l}5159(59.4 \\
58.1-60.8)\end{array}$ & $\begin{array}{l}26141(48.9,48.1- \\
49.7)\end{array}$ & $\begin{array}{l}201.5 \\
(P< \\
0.001)\end{array}$ \\
\hline \multicolumn{8}{|l|}{ Family Planning Messaging Received } \\
\hline Heard/saw FP message via TV in recent months? & $\begin{array}{l}8709 \\
(13.9)\end{array}$ & $\begin{array}{l}679(19.4 \\
17.7- \\
21.3)\end{array}$ & $\begin{array}{l}8030(13.9 \\
13.3-14.5)\end{array}$ & $\begin{array}{l}45.9 \\
(P< \\
0.001)\end{array}$ & $\begin{array}{l}1466(17.5 \\
16.4-18.6)\end{array}$ & $7243(13.3,12.8-13.9)$ & $\begin{array}{l}65.6(P \\
< \\
0.001)\end{array}$ \\
\hline Heard FP message on radio in recent months? & $\begin{array}{l}16061 \\
(26.2)\end{array}$ & $\begin{array}{l}1085 \\
(29.6 \\
27.6- \\
31.6)\end{array}$ & $\begin{array}{l}14976 \\
(26.1, \\
25.4-26.8)\end{array}$ & $\begin{array}{l}12.1 \\
(P= \\
0.001)\end{array}$ & $\begin{array}{l}2422(29.2 \\
27.8-30.5)\end{array}$ & $\begin{array}{l}13639(25.7,25.0- \\
26.5)\end{array}$ & $\begin{array}{l}26.8(P \\
< \\
0.001)\end{array}$ \\
\hline Read FP message in periodical in recent months? & $\begin{array}{l}4789 \\
(7.8)\end{array}$ & $\begin{array}{l}497(13.5, \\
12.0- \\
15.0)\end{array}$ & $\begin{array}{l}4292(7.6 \\
7.2-8.0)\end{array}$ & $\begin{array}{l}93.5 \\
(\mathrm{P}< \\
0.001)\end{array}$ & $\begin{array}{l}614(7.5 \\
6.7-8.3)\end{array}$ & $4175(7.8,7.4-8.2)$ & $\begin{array}{l}0.691 \\
(P= \\
0.406)\end{array}$ \\
\hline $\begin{array}{l}\text { Proportion who had visited a health facility in last } \\
\text { year }\end{array}$ & $\begin{array}{l}32654 \\
(53.0)\end{array}$ & $\begin{array}{l}2224 \\
(61.5 \\
59.4- \\
63.6)\end{array}$ & $\begin{array}{l}30340 \\
(52.6, \\
51.8-53.3)\end{array}$ & $\begin{array}{l}62.6 \\
(P< \\
0.001)\end{array}$ & $\begin{array}{l}5485(64.5 \\
63.2-65.8)\end{array}$ & $\begin{array}{l}27079(51.1,50.4- \\
51.9)\end{array}$ & $\begin{array}{l}350.1 \\
(P< \\
0.001)\end{array}$ \\
\hline
\end{tabular}




\begin{tabular}{|c|c|c|c|c|c|c|c|}
\hline $\begin{array}{l}\text { Family Planning Knowledge and Messaging } \\
\text { Variables }\end{array}$ & $\begin{array}{l}n= \\
62882\end{array}$ & $\begin{array}{l}\text { HIV } \\
\text { Positive, n } \\
=3663\end{array}$ & $\begin{array}{l}\text { HIV } \\
\text { Negative, } \\
\mathrm{n}=59219\end{array}$ & $\begin{array}{l}\chi^{2} \\
\text { (P- } \\
\text { Value) }\end{array}$ & $\begin{array}{l}\text { History of } \\
\text { Terminated } \\
\text { Pregnancy, } \\
\mathrm{n}=8650\end{array}$ & $\begin{array}{l}\text { No History of } \\
\text { Terminated Pregnancy, } \\
\mathrm{n}=\mathbf{5 4 2 3 2}\end{array}$ & $\begin{array}{l}\chi^{2} \\
\text { (P- } \\
\text { Value) }\end{array}$ \\
\hline $\begin{array}{l}\text { Out of those who had visited a health facility, how } \\
\text { many recalled being given FP info by provider? }\end{array}$ & $\begin{array}{l}9754 \\
(30.7)\end{array}$ & $\begin{array}{l}878(40.7 \\
38.1- \\
43.2)\end{array}$ & $\begin{array}{l}8876(30.0 \\
29.2-30.8)\end{array}$ & $\begin{array}{l}72.0 \\
(P< \\
0.001)\end{array}$ & $\begin{array}{l}1660(31.2 \\
29.6-32.7)\end{array}$ & $8094(30.6,29.8-31.5)$ & $\begin{array}{l}0.412 \\
(P= \\
0.521)\end{array}$ \\
\hline
\end{tabular}

The percentage of $\mathrm{HIV}+$ women knowing 'at least one modern method' of $\mathrm{FP}(97.7 \%, \mathrm{Cl}=96.9-98.3)$ was significantly higher $(\mathrm{p}<0.001)$ than the percentage of HIV- women that knew at least one modern method $(91.8 \%, \mathrm{Cl}=91.4-92.2)$. Likewise, the percentage of HTP women that knew at least one modern method $(96.0 \%, \mathrm{Cl}=95.5-96.5)$ was significantly higher $(\mathrm{p}<0.001)$ than the percentage of NHTP women that knew a modern method $(91.4 \%, \mathrm{Cl}=91.0-91.9)$. The percentage of $\mathrm{HIV}+$ women with knowledge of the pill $(93.1 \%, \mathrm{Cl}=91.7-94.2)$ was higher $(\mathrm{p}<0.001)$ than $\mathrm{HIV}$ - women with knowledge of the pill $(79.2 \%$, $\mathrm{Cl}=$ 78.6-79.9). A larger, statistically significant proportion of HIV + as opposed to HIV- women knew about all other categories of modern FP as well. The knowledge advantage for HIV + women was 23.8 percentage points (PP) for IUD $(p<0.001)$; 14.3 PP for injectables $(p<0.001)$; 12.1 PP for male condoms ( $<$ $0.001) ; 26.0$ for female condoms ( $p<0.001) ; 12.1$ for female sterilization $(p<0.001) ; 11.3$ for male sterilization $(p<0.001)$; and 26.3 for implants $(p<0.001)$.

The trend for modern FP knowledge was similar for HTP versus NHTP women. The only exception was knowledge of male sterilization, of which there was no statistical difference $(p=0.200)$. However, HTP as opposed to NHTP women had statistically significant higher knowledge levels for all other categories, with an advantage of 8.6 PP for knowledge of the pill ( $p<0.001)$, 7.1 PP for IUD ( $<<0.001)$; 9.7 PP for injectables $(p<0.001) ; 5.5$ for male condoms ( $<<0.001) ; 5.9$ for female condoms ( $p<0.001) ; 7.2$ for female sterilization $(p<0.001)$; and 5.1 for implants $(p<0.001)$. Concerning FP messaging, out of HIV + women that had visited a healthcare facility, $40.7 \%(\mathrm{Cl}=38.1-43.2)$ recalled being given FP information by a provider, which was higher $(\mathrm{p}<0.001)$ than $\mathrm{HIV}$ - women $(30.0 \%, \mathrm{Cl}=29.2-30.8)$. This trend was not present for HTP versus NHTP women $(\mathrm{p}=0.521)$. The percentage of HIV + women that recalled seeing a FP message on TV $(19.4 \%, \mathrm{Cl}=17.7-21.3)$ was higher $(p<0.001)$ than HIV- women $(13.9 \%, \mathrm{Cl}=13.3-14.5)$. The percentage of HTP women that recalled receiving a FP message via TV $(17.5 \%, 16.4-18.6)$ was higher $(p<0.001)$ than NHTP women $(13.3 \%, \mathrm{Cl}=12.8-13.9)$. The prevalence of HIV + women that heard a radio FP message $(29.6 \%, \mathrm{Cl}=27.6-31.6)$ was higher $(\mathrm{p}=0.001)$ than HIV- women $(26.1 \%, \mathrm{Cl}=25.4-26.8)$. The prevalence of HTP women who heard a radio FP message $(29.2 \%, \mathrm{Cl}=27.8-30.5)$ was higher $(\mathrm{p}<0.001)$ than NHTP women $(25.7 \%, \mathrm{Cl}=25.0-26.5)$. The percentage of HIV + women that read a FP message in a periodical $(13.5 \%, \mathrm{Cl}=12.0-15.0)$ was higher $(\mathrm{p}<0.001)$ than HIV- women $(7.6 \%, \mathrm{Cl}=7.2-8.0)$. This trend for periodical messaging was not present for HTP versus NHTP women ( $\mathrm{p}=0.406)$.

\section{Logistic Regression Results}

Table 5 shows the outcomes of the logistic regression analyses in terms of AORs, $95 \%$ Cls, and p-values. For modern methods of FP, HIV + as opposed to HIVwomen demonstrated higher odds of knowing 'at least one modern method' of $\mathrm{FP}(\mathrm{AOR}=1.54, \mathrm{Cl}=1.12-2.12)$, $\mathrm{knowing}$ the pill $(\mathrm{AOR}=1.39, \mathrm{Cl}=1.15-1.68)$, knowing the IUD $(A O R=1.23, \mathrm{Cl}=1.10-1.39)$, injectables $(A O R=1.33, \mathrm{Cl}=1.09-1.62)$, male condoms $(\mathrm{AOR}=1.85, \mathrm{Cl}=1.46-2.34)$, female condoms $(\mathrm{AOR}=$ $1.53, \mathrm{Cl}=1.33-1.76)$, and implants $(\mathrm{AOR}=1.44, \mathrm{Cl}=1.24-1.67)$. $\mathrm{HIV}+$ women did not have higher odds of knowing female sterilization $(A O R=1.01, \mathrm{Cl}=0.91-$ 1.12) or male sterilization ( $A O R=1.00, \mathrm{Cl}=0.89-1.11)$. HTP as opposed to NHTP women had higher odds of knowing 'at least one modern method' of $F P$ $(A O R=1.71, C l=1.47-1.98)$, knowing the pill $(A O R=1.50, C l=1.37-1.65)$, IUD $(A O R=1.19, C l=1.11-1.28)$, injectables $(A O R=1.60, C l=1.45-1.75)$, male condoms $(A O R=1.42, \mathrm{Cl}=1.28-1.58)$, female condoms $(A O R=1.22, \mathrm{Cl}=1.13-1.33)$, female sterilization $(A O R=1.22, \mathrm{Cl}=1.14-1.31)$, and implants $(A O R=$ $1.21, \mathrm{Cl}=1.12-1.31)$. HTP women did not demonstrate higher odds of knowing about male sterilization $(\mathrm{AOR}=0.97, \mathrm{Cl}=0.90-1.04)$. HIV + women that had visited a health facility in the previous 12 months, compared to HIV-women, showed higher odds of recalling having been given FP information by a provider $(A O R=1.23, \mathrm{Cl}=1.08-1.39)$. HTP as opposed to NHTP women that visited a healthcare facility did not demonstrate higher odds of recalling having been given $\mathrm{FP}$ information ( $\mathrm{AOR}=0.93, \mathrm{Cl}=0.85-1.01)$. $\mathrm{HIV}+$ women did not show higher odds of receiving a $\mathrm{FP}$ message from $\mathrm{TV}(\mathrm{AOR}=1.10, \mathrm{Cl}=0.95-1.27)$, radio $(A O R=0.93, C l=0.83-1.04)$, or a periodical $(A O R=0.97, C l=0.83-1.13)$. HTP women did show higher odds of receiving a $F P$ message from $T V(A O R=$ $1.13, \mathrm{Cl}=1.03-1.25)$ and radio $(\mathrm{AOR}=1.18, \mathrm{Cl}=1.09-1.26)$, but not from a periodical $(\mathrm{AOR}=0.92, \mathrm{Cl}=0.80-1.06)$. 
Table 5

Predicting family planning knowledge and messaging variables with HIV and pregnancy termination status using multivariate logistic regression

Outcome Variables

HIV Positive

P. History of Terminated

P-

Value Pregnancy

Value

Adjusted Odds Ratio Adjusted Odds Ratio

$(95 \% \mathrm{Cl})$

(95\% Cl)

Knows at least one modem method of FP?

$1.54(1.12-2.12) \quad 0.008 \quad 1.71(1.47-1.98)$

\section{Modern Methods of FP}

Knowledge about Pill for FP?

$1.39(1.15-1.68)$

$0.001 \quad 1.50(1.37-1.65)$

Knowledge about IUD for FP?

$1.23(1.10-1.39)$

$<$

$1.19(1.11-1.28)$

Know about FP injectables?

$1.33(1.09-1.62)$

$0.0051 .60(1.45-1.75)$

$<$

0.001

Know about male condoms for FP?

$1.85(1.46-2.34)$

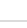

Know about female condoms for FP?

$1.53(1.33-1.76)$

$<$

(1.53

$<$

Know about female sterilization for FP?

$1.01(0.91-1.12)$

0.001

$1.42(1.28-1.58)$

$<$

0.001

Know about male sterilization for FP?

$1.00(0.89-1.11)$

$0.81 \quad 1.22(1.14-1.31)$

$1.44(1.24-1.67)$

$0.94 \quad 0.97(0.90-1.04)$

$1.21(1.12-1.31)$

0.001

$<$

0.001

Know about implants or Norplant for FP?

0.001

\section{Traditional Methods of FP}

Know about withdrawal for FP?

$1.15(1.03-1.28)$

0.01

$1.33(1.24-1.43)$

$<$

$<.001$

Know about rhythm method for FP?

$1.15(1.03-1.27)$

0.01

$1.21(1.13-1.30)$

\section{Family Planning Messaging Received}

Of those who visited a health facility in last 12 months, were you given FP information by a provider?

Heard/saw FP message via TV in recent months?

\begin{tabular}{llll}
$1.23(1.08-1.39)$ & 0.001 & $0.93(0.85-1.01)$ & 0.08 \\
\hline $1.10(0.95-1.27)$ & 0.22 & $1.13(1.03-1.25)$ & 0.01 \\
$0.93(0.83-1.04)$ & 0.20 & $1.18(1.09-1.26)$ & $<$ \\
$0.97(0.83-1.13)$ & 0.71 & $0.92(0.80-1.06)$ & 0.001
\end{tabular}

Heard FP message on radio in recent months?

Read FP message in periodical in recent months?

$0.97(0.83-1.13)$

0.71

$<$

0.001

0.35

Notes: Major predicting variables in the logistic regressions are HIV status (HIV negative is the reference group) and if the respondent ever experienced a terminated pregnancy (never experiencing pregnancy termination is the reference group). Covariates controlled in the logistic regression models are: country, age (continuous), urban/rural status, education level, wealth index quintile, whether or not they are currently working, electricity in household mobile phone ownership, current marital status, and whether the respondent considers herself to be the primary FP decider in the family.

\section{Discussion}

The primary goal of this analysis was to determine if HIV + versus HIV- women, and HTP versus NHTP women in fragile regions would exhibit differing FP knowledge profiles and/or report receiving FP information at different rates. For every category of modern FP knowledge, HIV + women demonstrated a higher level of awareness when compared to HIV- women in initial tests of independence. When covariates were controlled in the multivariate logistic regression models, HIV + women did not show higher odds of knowing about female or male sterilization; but still demonstrated higher odds of knowing about all other categories of modern FP. Likewise, HTP women demonstrated higher knowledge of every modern method of FP except for male sterilization in tests of independence. In the logistic regression models, HTP women showed higher odds of knowing about every category except for again, male sterilization. It would have been reasonable to expect that HIV + women would show higher knowledge of FP methods that also have sexually transmitted infection (STI) prevention capabilities (e.g., male and female condoms). However, the magnitude of the knowledge difference between HIV + and HIV- women was similar for several FP methods that had no STI prevention capabilities (e.g., the pill and implants). In other words, the Cls of the AORs for knowledge of FP methods with and without STI prevention capabilities overlapped. With respect to messaging, HIV + women were more likely than HIV- women to recall being given FP information by a provider at a recent visit to a healthcare facility. However, despite the fact that like HIV + women, HTP women are at an increased risk of future pregnancy complications [64-71], they were not more likely than NHTP women to be given FP information at a recent healthcare visit. HTP women did however exhibit higher odds than NHTP women of recalling encountering FP messaging via TV and radio. Since TV and radio messaging could not have been targeted in a way that would have resulted in HTP women having higher environmental exposure to the messages than NHTP women, it is possible that HTP women's past pregnancy experiences sensitized them to subsequent information related to that experience, making them more likely to notice the messaging 
and/or remember having been exposed to it. But, as establishing that was not the goal of this research, it would need to be explored in further specialized studies.

Although the primary focus of this analysis was not to compare countries, it is important to note that Burundi, Malawi, and Zimbabwe had committed to the United Nations Foundation's Family Planning 2020 (FP2020) initiative several years prior to the DHS being carried out in those countries; whereas Cameroon, DRC, Guinea, Cote d'Ivoire, and Niger either committed to FP2020 in the same year in which the DHS was carried out or years after [72]. The time prior to implementation of the DHS with which institutions in Burundi, Malawi, and Zimbabwe had to implement FP initiatives as a result of their commitment to FP2020 could have impacted the results for these countries. As of August 2021, Angola has not committed to FP2020.

When country of residence, HIV status, and HTP status were used to categorize respondents, the percentage in each category reporting knowledge of 'at least one modern method' of FP was relatively high. However, results indicated that relying on this metric alone was misleading with respect to holistically assessing FP knowledge, since the percentage of respondents with knowledge of each individual FP method was highly variable. For example, over $85 \%$ of respondents from Niger reported knowledge of at least one modern method, but only $18.3 \%$ and $50.8 \%$ had knowledge of IUDs and male condoms, respectively. Over $95 \%$ of HIV + women knew at least one modern method, but only $41.7 \%$ knew of male sterilization. Likewise, the percentage of respondents knowing at least one modern method was above $90 \%$ in HIV-, HTP, and NHTP women; but only about half of HIV- women knew about IUDs, $60.8 \%$ of HTP women knew about implants, and $61.1 \%$ of NHTP women knew about female condoms. Therefore, researchers assessing FP awareness are encouraged to not only focus on the percentage of women knowing at least one modern method, but to also take heed of the levels of awareness of specific FP subcategories, which will provide a more precise understanding of where gaps in FP knowledge in their populations of interest exist.

Knowledge and awareness, along with the provision, availability, and positive attitudes about FP are prerequisites to an individual ultimately using contraception. In one of the few studies that looked at FP in FCS, low use of contraceptives by women in a union was associated with low prevalence of FP knowledge in six local FCS regions in Sudan, Uganda, and the DRC [47]. Beyond the connection between FP knowledge and subsequent contraception use, one should also take into account the trends of demand for contraception not satisfied in countries within our sample when considering this study's implications. Slaymaker et al. [5] showed that between 1995 and 2018, Burundi, Malawi, Niger, and Zimbabwe demonstrated a decrease in the proportion of women who were sexually active and needing, but not using modern contraception. In Cameroon and the DRC there was no notable change in this proportion, while in Guinea and Cote d'Ivoire the proportion of women who were sexually active and needing, but not using modern contraception increased [5]. Angola was not included in their analysis [5]. While there is a degree of good news in the fact that this proportion decreased over time in Burundi, Malawi, Niger, and Zimbabwe, the most recently measured proportion of women who were sexually active and needing, but not using contraception in the countries within our sample, with the exception of Zimbabwe, were still extremely high. Moreira et al. [33] found that in Guinea (2012), 75.7\% of the demand for contraception was not satisfied. This percentage was 70.7\% in Angola (2015), 58.0\% in Cote d'Ivoire (2011), 56.4\% in the DRC (2013), 53.5\% in Niger (2012), 50.9\% in Burundi (2016), 46.4\% in Cameroon (2011), 25.2\% in Malawi (2015), and 14.0\% in Zimbabwe (2015) [33]. For comparison purposes, this value was recently about 10\% in the USA [5] and $8.6 \%$ in Columbia [33]. Decreasing these percentages is crucial for improving equity and gender issues, and health in general of women, families, and communities. When creating FP educational interventions meant to contribute to that cause, it is important that women with certain reproductive and/or sexual health histories be targeted due to their increased risk of a variety of negative outcomes. With that in mind, it is encouraging that our study indicates that HIV + and HTP women in fragile regions seem to have been reached as evidenced by their higher levels of FP knowledge across most categories. However, the other side of this could be an indication that women that do not have easily recognizable reproductive or sexual health histories may not be proactively reached as much as necessary. In other words, women that do not fall into identifiable "at risk" categories may be falling through the cracks, thereby perpetuating the cycle that results in women experiencing outcomes like HIV diagnoses or terminated pregnancies in the first place.

There are several limitations to this study. Although the target population was fragile regions, there were many fragile and/or conflict-affected countries not able to be included because DHS and/or AIS datasets were not available for them, or they did not meet other inclusion criteria outlined in the methods section. In fact, it is debatable how to even classify regions as FCS. We chose the WBG classification system since it is standardized and used as a guideline by a variety of international development stakeholders. Another limitation is that the classification of pregnancy termination by DHS included women who had miscarried, had a stillbirth, and/or undergone an abortion. These three events, and the circumstances leading up to them can be quite different. It would have been useful if these subcategories could have been analyzed separately. Lastly, this study was based on a cross-sectional survey with self-reported measures and therefore recall bias may have been an issue, temporality of observations and causation cannot be ascertained, and intercountry analyses are of limited value since the DHS surveys for each country were done in different years. Also, social desirability bias could have played a part in women misrepresenting their knowledge of FP. Despite these limitations, this study has several strengths. First, the DHS IPUMS data management tool allowed for a high degree of standardization, harmonization, and comparability between variables. The breadth of variables available in DHS enabled the inclusion of appropriate covariates in the regression models and allowed for robust descriptive analyses of HIV + and HTP prevalence across a variety of sociodemographic, reproductive, and sexual health subgroups, which alone provides useful information. This study is the only one to our knowledge that investigated trends and determinants of subcategories of FP knowledge along with FP messaging outcomes in a multi-country FCS target population using nationally representative surveys. Lastly, since DHS surveys are repeated approximately every five years, longitudinal follow-up analyses will be possible.

\section{Conclusion}

With this project we aimed to contribute to the reproductive health research base in fragile regions. The descriptive portion revealed that while the percentages of respondents reporting knowledge of at least one modern method of FP may be high, knowledge of specific subcategories of FP were extremely variable and in some cases very low. Increasing the awareness of a variety of modern methods of FP may enable women to access contraceptive methods that are better aligned with their personal desires. The inferential portion investigated predictors of FP knowledge and demonstrated the potential importance of breaking down analyses of FP knowledge by relevant medical histories of respondents. Further research is needed to clarify the mechanisms behind why we observed higher FP knowledge among HIV + and HTP women and why HIV + women were seemingly targeted with FP information more readily at health facility visits

Page 15/20 
than were HTP women; whereas HTP women were more likely to recall TV and radio FP messaging. Subsequent studies should also seek to determine which other population subgroups at risk for pregnancy complications are effectively being reached with FP education, and which are not. Better understanding not only trends but also predictors of FP knowledge can help practitioners improve FP outreach in more targeted and efficient ways by focusing resources on knowledge gaps and their causes. This efficiency is especially important in resource-poor and oftentimes unpredictable fragile settings.

\section{Abbreviations}

\section{FCS}

Fragile or Conflict-Affected Situations

FP

Family Planning

SSA

Sub-Saharan Africa

LMIC

Low and Middle-Income Country

DHS

Demographic and Health Surveys

NHTP

No History of Terminated Pregnancy

HTP

History of Terminated Pregnancy

AIS

AIDS Indicator Surveys

IRB

Institutional Review Board

DRC

Democratic Republic of Congo

WBG

World Bank Group

CPIA

Country Policy and Institutional Assessment

IUD

Intrauterine Device

Cl

Confidence Interval

AOR

Adjusted Odds Ratio

PP

Percentage Points

STI

Sexually Transmitted Infection

FP2020

United Nations Foundation Family Planning 2020 Initiative

\section{Declarations}

\section{Ethical approval and consent to participate}

This was a secondary analysis that involved using data from existing DHS and AIS datasets. No consent to participate was required given that there was no direct interaction with participants. As such, the Claremont Graduate University IRB exempted this study from further review.

\section{Consent for publication}

Not applicable.

\section{Availability of data and materials}

The data that support the findings of this study are available from DHS/AIS/IPUMS. However, permission must be granted by the DHS program to access and download the data. Therefore, the final dataset used in this study is available from the authors upon request but only after the interested party has also gained permission from DHS to access the individual country datasets that comprise our dataset.

\section{Competing interests}

Page $16 / 20$ 
The authors declare they have no competing interests.

\section{Funding}

No funding source was required for this study.

\section{Authors' contributions}

DJW conceived the initial idea and methodology of the study. DJW and BX performed data management and analysis, with BX providing methodological oversight and guidance. DJW prepared the first draft of manuscript, while DJW and BX worked together on subsequent revisions. Both authors approved the final version of the manuscript.

\section{Acknowledgements}

The authors would like to thank the DHS, AIS, and IPUMS programs for making this study possible. The authors would also like to thank Rielly Bingham, MPH and Fabiola Vatel, PhD for their reviews and valuable feedback.

\section{References}

1. The Organisation for Economic Co-operation and Development. States of Fragility 2018: Highlights [Internet]. OECD; 2018 [cited 2021 Jan 12 ]. 24 p. Available from: https://www.oecd.org/dac/conflict-fragility-resilience/docs/OECD\%20Highlights\%20documents_web.pdf.

2. Haar RJ, Rubenstein LS. Health in post-conflict and fragile states [Internet]. Washington, DC: United States Institute for Peace; 2012 Jan [cited 2021 Jan 14]. 16 p. Available from: https://www.usip.org/sites/default/files/SR_301.pdf.

3. Feldbaum H, Michaud J. Health diplomacy and the enduring relevance of foreign policy interests. PLoS Med. 2010 Apr 20; 7(4):e1000226.

4. Martineau T, McPake B, Theobald S, Raven J, Ensor T, Fustukian S, et al. Leaving no one behind: lessons on rebuilding health systems in conflict- and crisis-affected states. BMJ Glob Health. 2017 Jul 28; 2(2):e000327.

5. Slaymaker E, Scott RH, Palmer MJ, Palla L, Marston M, Gonsalves L, et al. Trends in sexual activity and demand for and use of modern contraceptive methods in 74 countries: a retrospective analysis of nationally representative surveys. Lancet Glob Health. 2020 Apr 1; 8(4):e567-e79.

6. Percival V, Richards E, MacLean T, Theobald S. Health systems and gender in post-conflict contexts: building back better? Confl Health. 2014 Oct 22; $8(19)$.

7. Haslegrave M. Sexual and reproductive health and rights in the sustainable development goals and the post-2015 development agenda: less than a year to go. Reprod Health Matters. 2014 Nov; 22(44):102-8.

8. Goodkind D, Lollock L, Choi Y, McDevitt T, West L. The demographic impact and development benefits of meeting demand for family planning with modern contraceptive methods. Glob Health Action. 2018; 11(1):1423861.

9. Canning D, Schultz TP. The economic consequences of reproductive health and family planning. Lancet. 2012 Jul 14; 380(9837):165-71.

10. Bornemisza O, Ranson MK, Poletti TM, Sondorp E. Promoting health equity in conflict-affected fragile states. Soc Sci Med. 2010 Jan; 70(1):80-8.

11. Woodward A, Sondorp E, Witter S, Martineau T. Health systems research in fragile and conflict-affected states: a research agenda-setting exercise. Health Res Policy Sys. 2016 Jul 21; 14(51).

12. Ranson K, Poletti T, Bornemisza O, Sondorp E. Promoting health equity in conflict-affected fragile states [Internet]. London School of Hygiene \& Tropical Medicine; 2007 Feb 3 [cited 2021 Jan 25]. Available from:

https://www.who.int/social_determinants/resources/csdh_media/promoting_equity_conflict_2007_en.pdf.

13. Newbrander W, Waldman R, Shepherd-Banigan M. Rebuilding and strengthening health systems and providing basic health services in fragile states. Disasters. 2011 Oct; 35(4):639-60.

14. Ahuka OL, Chabikuli N, Ogunbanjo GA. The effects of armed conflict on pregnancy outcomes in the Congo. Int J of Gynaecol Obstet. 2004 Jan; 84(1):912.

15. Coghlan B, Brennan R, Ngoy P, Dofara D, Otto B, Stewart T. Mortality in the Democratic Republic of Congo: results from a nationwide survey [Internet]. International Rescue Committee; 2005 [cited 2021 Jan 19]. Available from: http://archive2.grip.org/bdg/pdf/g4231.pdf.

16. McGinn T, Casey SE, Purdin S, Marsh M. Reproductive health for conflict-affected people: policy, research and programmes [Internet]. London, UK: Humanitarian Practice Network at Overseas Development Institute; 2004 Apr [cited 2021 Jan 16]; No 45:[37 p.]. Available from:

https://www.researchgate.net/publication/242709948_Reproductive_health_for_conflict-affected_people_Policy_research_and_programmes.

17. Keasley J, Blickwedel J, Quenby S. Adverse effects of exposure to armed conflict on pregnancy: a systematic review. BMJ Glob Health. 2017 Nov 28 ; 2(4):e000377.

18. UNAIDS. UNAIDS data 2020 [Internet]. UNAIDS; 2020 Jul 6 [cited 2021 Feb 17]; [436 p.]. Available from:

https://www.unaids.org/sites/default/files/media_asset/2020_aids-data-book_en.pdf.

19. Spiegel PB, Bennedsen AR, Claass J, Bruns L, Patterson N, Yiweza D, et al. Prevalence of HIV infection in conflict-affected and displaced people in seven sub-Saharan African countries: a systematic review. Lancet. 2007 Jun 30; 369(9580):2187-95.

20. Spiegel PB. HIV/AIDS among conflict-affected and displaced populations: dispelling myths and taking action. Disasters. 2004 Sep; $28(3): 322-39$.

21. Omare D, Kanekar A. Determinants of HIV/AIDS in armed conflict populations. J Public Health Afr. 2011 Mar 1; 2(1):e9. 
22. Mock NB, Duale S, Brown LF, Mathys E, O'maonaigh HC, Abul-Husn NKL, et al. Conflict and HIV: a framework for risk assessment to prevent HIV in conflictaffected settings in Africa. Emerg Themes Epidemiol. 2004 Oct 29; 1(1):6.

23. Mills EJ, Singh S, Nelson BD, Nachega JB. The impact of conflict on HIV/AIDS in sub-Saharan Africa. Int J STD AIDS. 2006 Nov; $17(11): 713-7$.

24. Becker JU, Theodosis C, Kulkarni R. HIV/AIDS, conflict and security in Africa: rethinking relationships. J Int AIDS Soc. 2008 Sep 22; 11:3.

25. Iqbal Z, Zorn C. Violent conflict and the spread of HIV/AIDS in Africa. J Polit. 2010 Jan; 72(1):149-62.

26. Gruber J. Does conflict increase vulnerability to HIV infection? Issues for a research agenda. Afr J AIDS Res. 2006 May; 5(1):41-8.

27. Blencowe H, Cousens S, Jassir FB, Say L, Chou D, Mathers C, et al. National, regional, and worldwide estimates of stillbirth rates in 2015 , with trends from 2000: a systematic analysis. Lancet Glob Health. 2016 Jan 18; 4(2):e98-e108.

28. Lawn JE, Blencowe H, Waiswa P, Amouzou A, Mathers C, Hogan D, et al. Stillbirths: rates, risk factors, and acceleration towards 2030. Lancet. 2016 Feb 6; 387(10018):587-603.

29. World Health Organization. Maternal, newborn, child and adolescent health and ageing [Internet]. WHO; c2021 [cited 2021 Jan 26$]$. Available from: https://www.who.int/data/maternal-newborn-child-adolescent-ageing/maternal-and-newborn-data.

30. Deitch J, Stark L. Adolescent demand for contraception and family planning services in low- and middle-income countries: a systematic review. Glob Public Health. 2019 Sep; 14(9):1316-34.

31. Kalamar AM, Tunçalp Ö, Hindin MJ. Developing strategies to address contraceptive needs of adolescents: exploring patterns of use among sexually active adolescents in 46 low- and middle-income countries. Contraception. 2018 Jul; 98(1):36-40.

32. Li Z, Patton G, Sabet F, Zhou Z, Subramanian SV, Lu C. Contraceptive use in adolescent girls and adult women in low- and middle-income countries. JAMA Netw Open. 2020 Feb 19; 3(2):e1921437.

33. Moreira LR, Ewerling F, Barros AJD, Silveira MF. Reasons for nonuse of contraceptive methods by women with demand for contraception not satisfied: an assessment of low and middle-income countries using demographic and health surveys. Reprod Health. 2019 Oct 11; $16(1): 148$.

34. Wulifan JK, Brenner S, Jahn A, De Allegri M. A scoping review on determinants of unmet need for family planning among women of reproductive age in low and middle income countries. BMC Womens Health. 2016 Jan 15; 16(1):2.

35. Amjad S, MacDonald I, Chambers T, Osornio-Vargas A, Chandra S, Voaklander D, et al. Social determinants of health and adverse maternal and birth outcomes in adolescent pregnancies: a systematic review and meta-analysis. Paediatr Perinat Epidemiol. 2019 Jan; 33(1):88-99.

36. Kebede AS, Muche AA, Alene AG. Factors associated with adverse pregnancy outcome in Debre Tabor town, northwest Ethiopia: a case control study. BMC Res Notes. 2018 Nov 19; 11(1):820.

37. Kramer MS. The epidemiology of adverse pregnancy outcomes: an overview. J Nutr. 2003 May; 133(5 Suppl 2):1592S-6S.

38. Kurth F, Bélard S, Mombo-Ngoma G, Schuster K, Adegnika AA, Bouyou-Akotet MK, et al. Adolescence as risk factor for adverse pregnancy outcome in Central Africa-a cross-sectional study. PLoS One. 2010 Dec 20; 5(12):e14367.

39. Dias SS, Mbofana F, Cassy SR, Dias S, Augusto GF, Agadjanian V, et al. Estimating risk factors for HIV infection among women in Mozambique using population-based survey data. Afr J AIDS Res. 2018 Mar; 17(1):62-71.

40. Drain PK, Smith JS, Hughes JP, Halperin DT, Holmes KK. Correlates of national HIV seroprevalence: an ecologic analysis of 122 developing countries. J Acquir Immune Defic Syndr. 2004 Apr 1; 35(4):407-20.

41. Fox AM. The social determinants of HIV serostatus in sub-Saharan Africa: an inverse relationship between poverty and HIV? Public Health Rep. 2010 JulAug; 125 Suppl 4(Suppl 4):16-24.

42. Wojcicki JM. Socioeconomic status as a risk factor for HIV infection in women in East, Central and Southern Africa: a systematic review. J Biosoc Sci. 2005 Jan; 37(1):1-36.

43. Gelaw YA, Magalhães RJS, Assefa Y, Williams G. Spatial clustering and socio-demographic determinants of HIV infection in Ethiopia, 2015-2017. Int J Infect Dis. 2019 May; 82:33-39.

44. Fleming PJ, Shakya H, Farron M, Brooks MI, Lauro G, Levtov RG, et al. Knowledge, attitudes, and practices related to family planning and gender equity among husbands of adolescent girls in Niger. Glob Public Health. 2020 May; 15(5):666-77.

45. Mustafa G, Azmat SK, Hameed W, Ali S, Ishaque M, Hussain W, et al. Family planning knowledge, attitudes, and practices among married men and women in rural areas of Pakistan: findings from a qualitative need assessment study. Int J Reprod Med. 2015; 2015:190520.

46. Salisbury P, Hall L, Kulkus S, Paw MK, Tun NW, Min AM, et al. Family planning knowledge, attitudes and practices in refugee and migrant pregnant and post-partum women on the Thailand-Myanmar border - a mixed methods study. Reprod Health. 2016 Aug 19; $13(1): 94$.

47. McGinn T, Austin J, Casey SE, Schlecht J, Anfinson K, Sharer M, et al. Family planning in conflict: results of cross-sectional baseline surveys in three African countries. Confl Health. 2011 Jul 13; 5(11).

48. The DHS Program. Demographic and Health Surveys [Internet]. Rockville, MD: ICF; c2021 [cited 2021 Jan 5]. Available from: https://dhsprogram.com/.

49. Corsi DJ, Neuman M, Finlay JE, Subramanian SV. Demographic and health surveys: a profile. Int J Epidemiol. 2012 Dec; $41(6): 1602-13$.

50. The DHS Program. AIS overview [Internet]. Rockville, MD: ICF; c2021 [cited 2021 Jan 5]. Available from: https://dhsprogram.com/methodology/surveytypes/ais.cfm.

51. IPUMS Global Health. Harmonized international survey data on maternal, child, and reproductive health [Internet]. University of Minnesota; c2021 [cited 2021 Jan 5]. Available from: https://globalhealth.ipums.org/

52. ICF International Inc. The demographic and health surveys (DHS) program [Internet]. ICF International; c2021 [cited 2021 Jan 5]. Available from: https://www.icf.com/clients/health/demographic-health-surveys-technical-assistance.

Page $18 / 20$ 
53. Boyle EH, King M, Sobek M. IPUMS demographic and health surveys. Version 8 [dataset]. Minneapolis, MN: IPUMS and ICF; c2020. [cited 2020 Aug 10]. Available from: https://doi.org/10.18128/D080.V8.

54. The World Bank Group. Classification of fragile and conflict-affected situations [Internet]. World Bank Group; c2021 [cited 2021 Jan 15]. Available from: https://www.worldbank.org/en/topic/fragilityconflictviolence/brief/harmonized-list-of-fragile-situations.

55. The World Bank Group. CPIA criteria 2017 [Internet]. World Bank Group; 2017 Sep 1 [cited 2021 Jan 15]. Available from: http://pubdocs.worldbank.org/en/203511467141304327/CPIA-Criteria-2017v2.pdf.

56. ACLED. The armed conflict location \& event data project [Internet]. ACLED; c2021 [cited 2021 Jan 15]. Available from: https://acleddata.com/\#/dashboard.

57. UCDP. Uppsala conflict data program: department of peace and conflict research [Internet]. Uppsala, Sweden: Uppsala University; c2021 [cited 2021 Jan 15]. Available from: https://ucdp.uu.se/exploratory.

58. Croft TN, Marshall AMJ, Allen CK, et al. Guide to DHS statistics: DHS-7 (version 2). [Internet] Rockville, MD: ICF; 2020 May [cited 2021 Jan 23]. Available from: https://www.dhsprogram.com/pubs/pdf/DHSG1/Guide_to_DHS_Statistics_DHS-7_v2.pdf.

59. Beier ME, Ackerman PL. Determinants of health knowledge: an investigation of age, gender, abilities, personality, and interests. J Pers Soc Psychol. 2003 Feb; 84(2):439-48.

60. Freimuth VS. The chronically uninformed: closing the knowledge gap in health. In: Ray EB, Donohew L, editors. Communication and health: systems and applications. Hillsdale, NJ: Lawrence Erlbaum Associates; 1990. p. 171-186.

61. Neisser U, Boodoo G, Bouchard Jr TJ, Boykin AW, Brody N, Ceci SJ, et al. Intelligence: knowns and unknowns. Am Psychol. 1996; 51(2):77-101.

62. Sandman D, Simantov E, An C. Out of touch: American men and the health care system [Internet]. New York: The Commonwealth Fund; 2000 Mar [cited 2021 Feb 7]: [60 p.]. Available from:

https://www.commonwealthfund.org/sites/default/files/documents/_media_files_publications_fund_report_2000_mar_out_of_touch_american_men_a

63. IBM. IBM SPSS software [Internet]. IBM; c2021 [cited 2021 Jan 3]. Available from: https://www.ibm.com/analytics/spss-statistics-software.

64. Knudsen UB, Hansen V, Juul S, Secher NJ. Prognosis of a new pregnancy following previous spontaneous abortions. Eur J Obstet Gynecol Reprod Biol. 1991 Mar 21; 39(1):31-6.

65. Regan L, Braude PR, Trembath PL. Influence of past reproductive performance on risk of spontaneous abortion. BMJ. 1989 Aug 26; 299(6698):541-5.

66. Risch HA, Weiss NS, Clarke EA, Miller AB. Risk factors for spontaneous abortion and its recurrence. Am J Epidemiol. 1988 Aug; 128(2):420-30.

67. Ticconi C, Pietropolli A, Specchia M, Nicastri E, Chiaramonte C, Piccione E, et al. Pregnancy-related complications in women with recurrent pregnancy loss: a prospective cohort study. J Clin Med. 2020 Sep 1; 9(9):2833.

68. Habib NA, Daltveit AK, Bergsjø P, Shao J, Oneko O, Lie RT. Maternal HIV status and pregnancy outcomes in northeastern Tanzania: a registry-based study. BJOG. 2008 Apr; 115(5):616-24.

69. Li H, Liu J, Tan D, Huang G, Zheng J, Xiao J, et al. Maternal HIV infection and risk of adverse pregnancy outcomes in Hunan province, China: a prospective cohort study. Medicine (Baltimore). 2020 Feb; 99(8):e19213.

70. Rollins NC, Coovadia HM, Bland RM, Coutsoudis A, Bennish ML, Patel D, et al. Pregnancy outcomes in HIV-infected and uninfected women in rural and urban South Africa. J Acquir Immune Defic Syndr. 2007 Mar 1; 44(3):321-8.

71. Onah HE, Obi SN, Agbata TA, Oguanuo TC. Pregnancy outcome in HIV-positive women in Enugu, Nigeria. J Obstet Gynaecol. 2007 Apr; $27(3)$ :271-4.

72. United Nations Foundation. FP family planning 2020 [Internet]. Washington, DC: United Nations Foundation; c2020 [cited 2021 Jan 6]. Available from: https://www.familyplanning2020.org/.

\section{Figures}




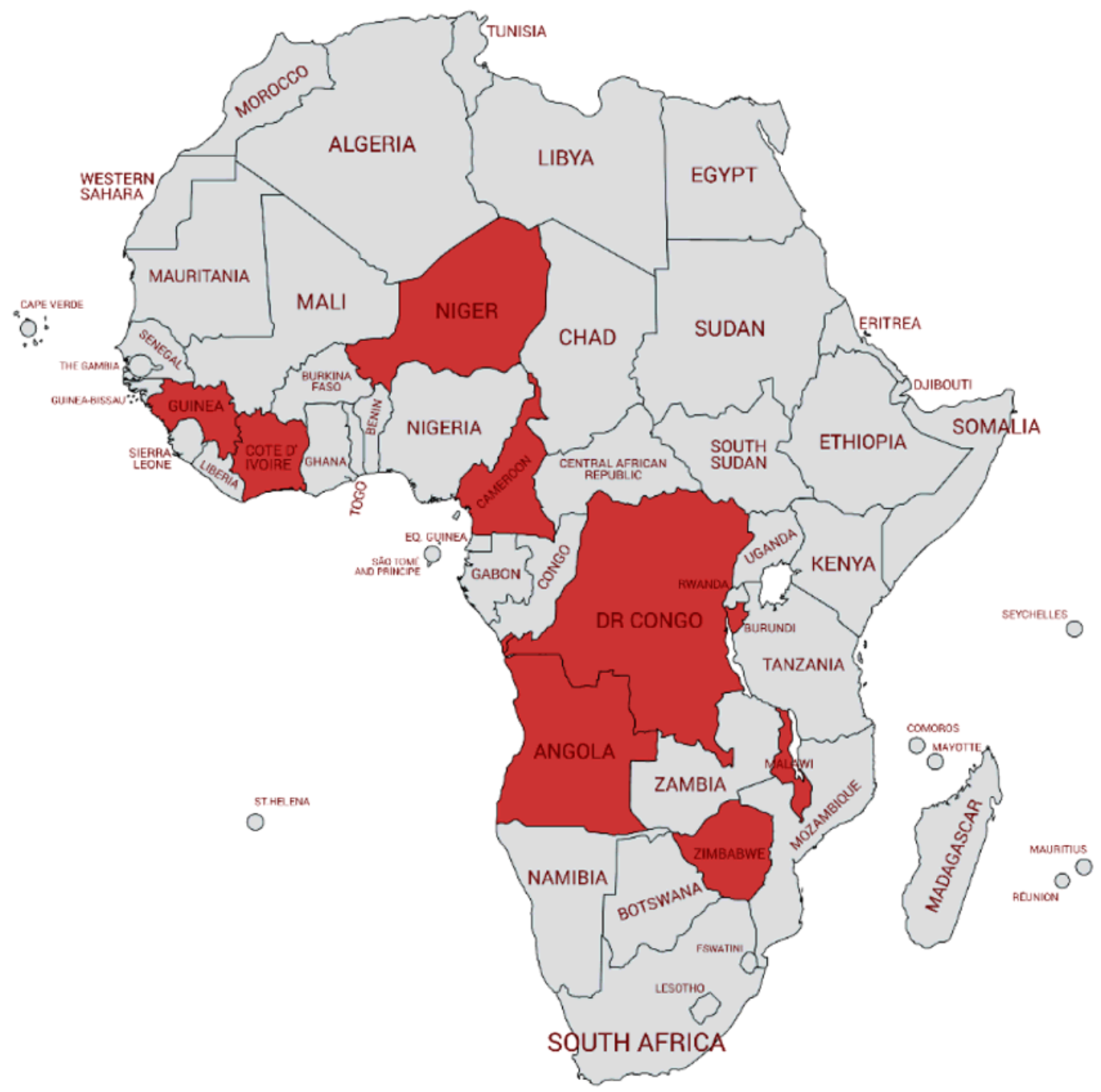

Figure 1

Geographic locations of included countries (red) 